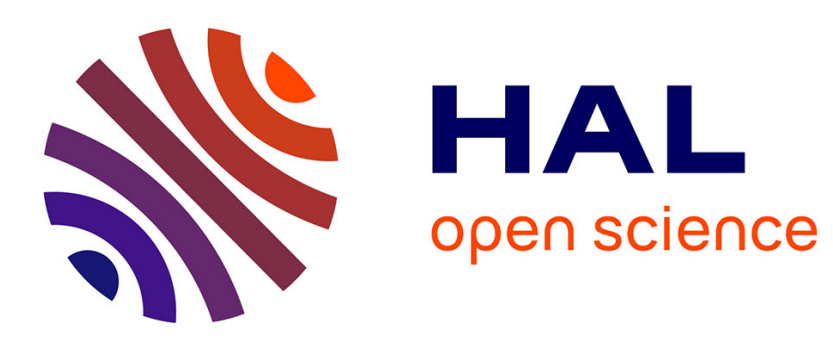

\title{
Synthese und Charakterisierung von binären Molekülverbindungen der Gruppe 15 Elemente mit P2As2-, PSb2-, PBi2-, PBi3- und P2Bi4-Gerüst
}

\author{
Carsten von Hänisch, Sven Stahl
}

\section{- To cite this version:}

Carsten von Hänisch, Sven Stahl. Synthese und Charakterisierung von binären Molekülverbindungen der Gruppe 15 Elemente mit P2As2-, PSb2-, PBi2-, PBi3- und P2Bi4-Gerüst. Journal of Inorganic and General Chemistry / Zeitschrift für anorganische und allgemeine Chemie, 2009, 635 (13-14), pp.2230. 10.1002/zaac.200900172 . hal-00507769

\section{HAL Id: hal-00507769 \\ https://hal.science/hal-00507769}

Submitted on 31 Jul 2010

HAL is a multi-disciplinary open access archive for the deposit and dissemination of scientific research documents, whether they are published or not. The documents may come from teaching and research institutions in France or abroad, or from public or private research centers.
L'archive ouverte pluridisciplinaire HAL, est destinée au dépôt et à la diffusion de documents scientifiques de niveau recherche, publiés ou non, émanant des établissements d'enseignement et de recherche français ou étrangers, des laboratoires publics ou privés. 


\section{Synthese und Charakterisierung von binären}

Molekülverbindungen der Gruppe 15 Elemente mit P2As2-, PSb2-, PBi2-, PBi3- und P2Bi4-Gerüst

\begin{tabular}{|r|l|}
\hline Journal: & Zeitschrift für Anorganische und Allgemeine Chemie \\
\hline Manuscript ID: & zaac.200900172.R1 \\
\hline Wiley - Manuscript type: & Research Report \\
\hline Date Submitted by the \\
Author: & 14-May-2009 \\
\hline Keywords: & $\begin{array}{l}\text { Arsenic, Antimony, Bismuth, Phosphorus, Ring Compounds } \\
\text { Nanotechnologie } \\
\text { Nanotechnologie }\end{array}$ \\
\hline
\end{tabular}

\section{scholarONE \\ Manuscript Central}


Synthese und Charakterisierung von binären Molekülverbindungen der Gruppe 15

Elemente mit $\mathbf{P}_{2} \mathrm{As}_{2}-$, $\mathbf{P S b}_{2-}, \mathbf{P B i}_{2^{-}}, \mathbf{P B i}_{3^{-}}$und $\mathbf{P}_{2} \mathbf{B i}_{4}$-Gerüst.

Synthesis and characterisation of binary Molecular compounds of the group 15

Elements with $\mathbf{P}_{2} \mathrm{As}_{2}, \mathrm{PSb}_{2}, \mathbf{P B i}_{2}, \mathbf{P B i}_{3}$ und $\mathbf{P}_{2} \mathrm{Bi}_{4}$ structures

Carsten von Hänisch*, und Sven Stahl

* PD Dr. C. von Hänisch

Institut für Nanotechnologie, Forschungszentrum Karlsruhe

Postfach 3640, D-76021 Karlsruhe

Telefax: 07247/82-6368

E-Mail: carsten.vonhaenisch@int.fzk.de

Bei der Redaktion eingegangen am

Keywords: Arsenic, Antimony, Bismuth, Phosphorus, Ring Compounds

\begin{abstract}
The reactions of $t \mathrm{BuPh}_{2} \mathrm{SiPLi}_{2}$ with $\left(\mathrm{Me}_{3} \mathrm{Si}_{2}\right)_{2} \mathrm{CHAsCl}_{2}$ in molar ratio $1: 1$ yields the compound $\left[\left(\mathrm{SiMe}_{3}\right)_{2} \mathrm{CHAsPSi} \mathrm{BuPh}_{2}\right]_{2}$ (1). The structure of $\mathbf{1}$ reveals the formation of a $\mathrm{As}_{2} \mathrm{P}_{2}$ ring compound. In contrast, the reactions of $t \mathrm{BuPh}_{2} \mathrm{SiPLi}_{2}$ with $\left(\mathrm{Me}_{3} \mathrm{Si}\right)_{2} \mathrm{CHSbCl}_{2}$ or $\left(\mathrm{Me}_{3} \mathrm{Si}\right)_{2} \mathrm{CHBiCl}_{2}$ yield the chloro bridged species $\left[t \mathrm{BuPh}_{2} \mathrm{SiP}\left\{\mathrm{SbClCH}\left(\mathrm{SiMe}_{3}\right)_{2}\right\}_{2}\right](2)$ and $\left[t \mathrm{BuPh}_{2} \mathrm{SiP}\left\{\mathrm{BiClCH}\left(\mathrm{SiMe}_{3}\right)_{2}\right\}_{2}\right]$ (3). Starting from $\mathrm{RMe}_{2} \mathrm{SiPLi}_{2}(\mathrm{R}=\mathrm{Thex}, \mathrm{Me})$ the compounds $\left[\mathrm{P}\left\{\mathrm{BiClCH}\left(\mathrm{SiMe}_{3}\right)_{2}\right\}_{3}\right]$ (4) and $\left[\mathrm{P}_{2}\left\{\mathrm{BiClCH}\left(\mathrm{SiMe}_{3}\right)_{2}\right\}_{4}\right]$ (5) were obtained from the reactions with $\left(\mathrm{Me}_{3} \mathrm{Si}\right)_{2} \mathrm{CHBiCl}_{2}$. The Compounds $\mathbf{1}-\mathbf{5}$ were characterised by single crystal X-Ray diffraction, elemental analysis as well as IR and NMR techniques.
\end{abstract}

\title{
Einleitung
}

Die Synthese von homonuklearen Ringen und Polycyclen der Gruppe-15-Elemente steht bereits seit langer Zeit im Focus der anorganischen Molekülchemie. ${ }^{[1-3]}$ Dabei konnten verschiedenste Molekülstrukturen realisiert werden. Die Palette reicht von kleinen Ringen wie z. B. $\mathrm{P}_{3} t \mathrm{Bu}_{3}$ [2] über größere Ringe bis zu polcylischen oder käfigartigen Spezies [3]. Unter 
den möglichen binären Verbindungen sind bisher in erster Linie die Phosphor-StickstoffHeterocyclen untersucht [4], während über Heterocyclen aus zwei verschiedenen schwereren Elementen der 5. Gruppe ( $\mathrm{P}, \mathrm{As}, \mathrm{Sb}, \mathrm{Bi})$ vergleichsweise wenig bekannt ist. $\mathrm{Zu}$ den beschriebenen Verbindungen dieses Typs gehört z. B. der Ring $\left(\mathrm{P}_{2} \mathrm{As}_{\mathrm{Bu}}\right)$, der bei der Reaktion von $t \mathrm{Bu}_{2} \mathrm{P}_{2} \mathrm{~K}_{2}$ mit $t \mathrm{BuAsCl}_{2}$ gebildet wird [5]. Desweiteren sind die bicyclischen Verbindungen $\left[\mathrm{As}_{2}(\mathrm{PAr})_{2}\right]$ and $\left[\mathrm{Sb}_{2}(\mathrm{PAr})_{2}\right]$ bekannt, die durch die Umsetzung von $\operatorname{ArP}\left(\mathrm{SiMe}_{3}\right) \mathrm{Li}\left(\mathrm{Ar}=2,4,6-(t \mathrm{Bu})_{3} \mathrm{C}_{6} \mathrm{H}_{2}\right)$ mit $\mathrm{Cp}^{*} \mathrm{E}^{\prime} \mathrm{Cl}_{2}\left(\mathrm{E}^{\prime}=\mathrm{As}, \mathrm{Sb}\right)$ erhalten wurden sowie die ebenfalls bicyclischen Verbindungen $\left[(t \mathrm{BuP})_{3} \mathrm{As}\right]_{2}$ und $\left[(t \mathrm{BuP})_{3} \mathrm{Sb}\right]_{2}$ [6]. Darüber hinaus wurden kürzlich die binären Käfige $\left[\mathrm{Sb}_{4}\left(\mathrm{PSiMe}_{2} \mathrm{Thex}\right)_{4}\right]$ und $\left[\mathrm{Sb}_{4}\left(\mathrm{AsSi}_{2} \mathrm{Pr}_{3}\right)_{4}\right]$ sowie das phosphanylsubstituierte Dibismuten [BiP( $\left.\left.\mathrm{Sit} \mathrm{BuPh}_{2}\right)_{2}\right]_{2}$ erhalten [7].

Wir berichten hier über die Synthesen und Kristallstrukturen der cyclischen Verbindung $\left[\left(\mathrm{SiMe}_{3}\right)_{2} \mathrm{CHAsPSit} \mathrm{BuPh}_{2}\right]_{2} \quad$ (1) sowie der chlorverbrückten Spezies $\left[t \mathrm{BuPh}_{2} \mathrm{SiP}\left\{\mathrm{SbClCH}\left(\mathrm{SiMe}_{3}\right)_{2}\right\}_{2}\right] \quad$ (2), $\quad\left[t \mathrm{BuPh}_{2} \mathrm{SiP}\left\{\mathrm{BiClCH}\left(\mathrm{SiMe}_{3}\right)_{2}\right\}_{2}\right] \quad$ (3), $\left[\mathrm{P}\left\{\mathrm{BiClCH}\left(\mathrm{SiMe}_{3}\right)_{2}\right\}_{3}\right]$ (4) und $\left[\mathrm{P}_{2}\left\{\mathrm{BiClCH}\left(\mathrm{SiMe}_{3}\right)_{2}\right\}_{4}\right]$ (5), die bei Reaktionen der Dilithiumphosphandiide $t \mathrm{BuPh}_{2} \mathrm{SiPLi}_{2}$, ThexMe $\mathrm{SiPLi}_{2}\left(\mathrm{Thex}=\mathrm{CMe}_{2} i \mathrm{Pr}\right.$ ) und $\mathrm{Me}_{3} \mathrm{SiPLi}_{2}$ mit $\mathrm{R}^{*} \mathrm{ECl}_{2}\left(\mathrm{E}=\mathrm{As}, \mathrm{Sb}, \mathrm{Bi} ; \mathrm{R}^{*}=\mathrm{CH}\left(\mathrm{SiMe}_{3}\right)_{2}\right)$ erhalten wurden (Schema 1).

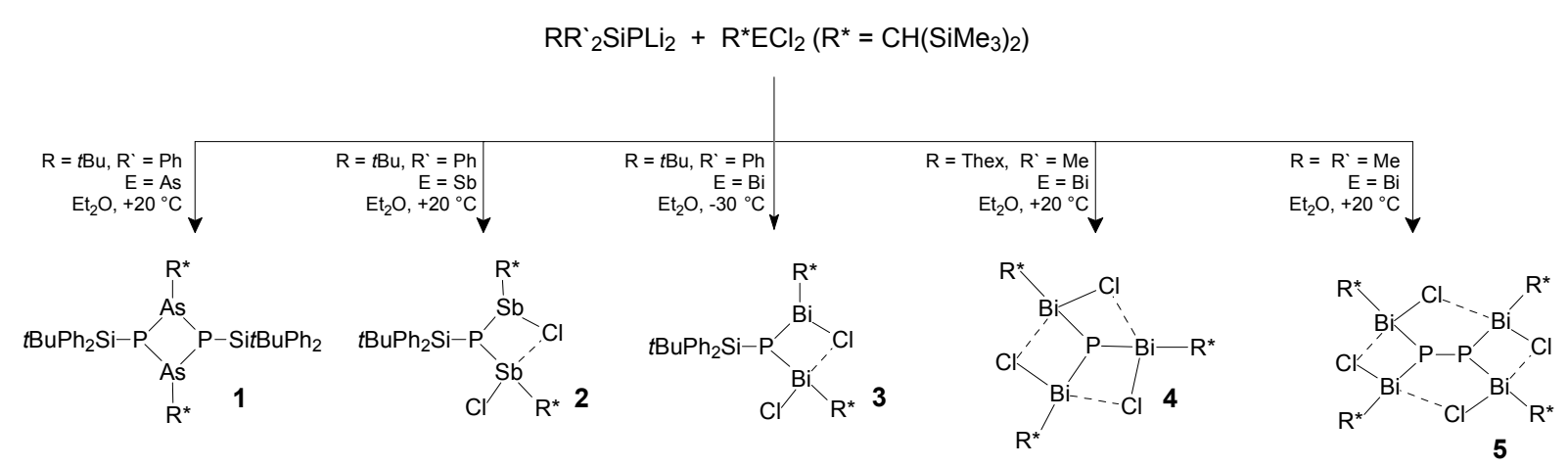

Schema 1 Darstellung der Verbindungen 1 - 5

\section{Ergebnisse und Diskussion}

Die Reaktion von $t \mathrm{BuPh}_{2} \mathrm{SiPLi}_{2}$ mit $\left(\mathrm{SiMe}_{3}\right)_{2} \mathrm{CHAsCl}_{2}$ in einem molaren Verhältnis von 1:1 führt zur Bildung von Verbindung 1, die durch Kühlen der Reaktionslösung auf $-35^{\circ} \mathrm{C}$ in Form von gelben Kristallen erhalten werden kann. 1 kristallisiert in der monoklinen Raumgruppe $P 2{ }_{1} / n$ und besteht aus einem $\mathrm{P}_{2} \mathrm{As}_{2}$-Vierring mit alternierender Elementfolge [9]. Die Phosphoratome weisen je einen exocyclischen Silylsubstituenten auf, jedes der Arsenatome bindet an einen $\mathrm{CH}\left(\mathrm{SiMe}_{3}\right)_{2}$-Rest. Der $\mathrm{P}_{2} \mathrm{As}_{2}$-Ring ist nicht planar sondern weist mit Faltungswinkeln von $149.2^{\circ}$ (entlang der P-P-Achse) und $122.3^{\circ}$ (entlang der As-As- 
Achse) eine schmetterlingsartige Faltung auf. Die P-As-Bindungen in $\mathbf{1}$ sind durchschnittlich 236.5 pm lang, die P-As-P- bzw. As-P-As-Winkel betragen durchschnittlich 81.6 bzw. $83.5^{\circ}$.

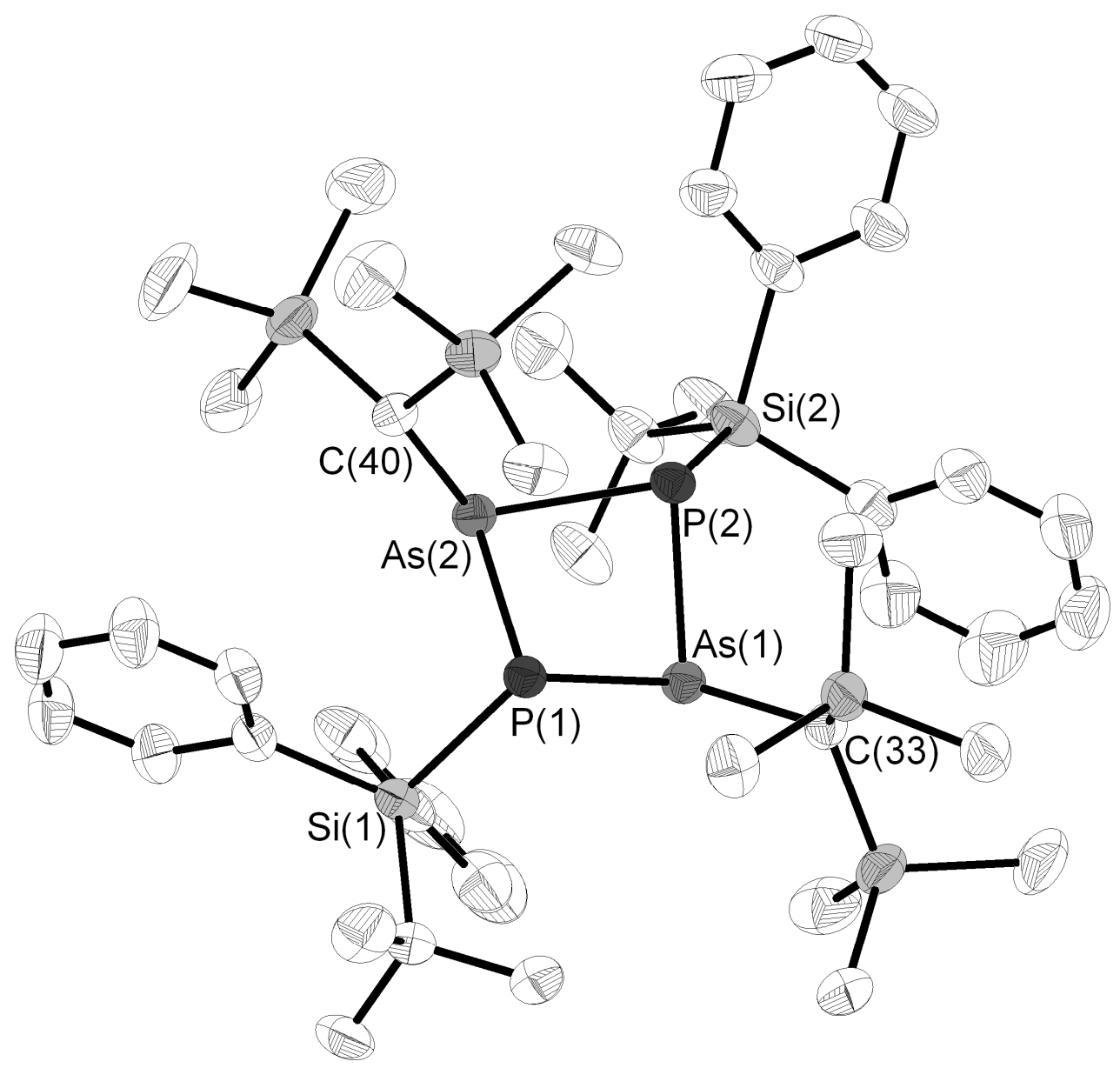

Abbildung 1 Molekülstruktur von 1 im Kristall (40\% Wahrscheinlichkeit für die Schwingungsellipsoide, die Wasserstoffatome sind nicht gezeigt).

Ausgesuchte Bindungslängen/pm und -winkel/ ${ }^{\circ}$ : As(1)-P(1) 237.3(1), As(2)-P(1) 235.3(1), $\operatorname{As}(1)-\mathrm{P}(2)$ 236.7(1), As(2)-P(2) 236.8(1), P(1)-Si(1) 227.8(1), P(2)-Si(2) 227.2(1), As(1)C(33) 202.4(3), As(2)-C(40) 201.2(3); As-P-As 83.47(3) und 83.68(3), P-As-P 81.36(3) und 81.75(3), Si(1)-P(1)-As(1) 104.33(4), Si(1)-P(1)-As(2) 96.63(4), Si(2)-P(2)-As(1) 99.61(4), $\quad \mathrm{Si}(2)-\mathrm{P}(2)-\mathrm{As}(2) \quad$ 108.98(4), $\quad$ C(33)-As(1)-P(1) 110.28(9), C(33)-As(1)-P(2) 103.05(9), C(40)-As(2)-P(1) 103.66(9), C(40)-As(2)-P(2) 109.28(7).

Das ${ }^{31}$ P-NMR-Spektrum von 1 zeigt ein Tripplet bei -97 ppm mit einer Kopplungskonstanten von $8 \mathrm{~Hz}$ zu den tertiären Protonen der $\mathrm{CH}\left(\mathrm{SiMe}_{3}\right)_{2}$-Gruppen. Im EI-Massenspektrum von 1 
wird der Molekülpeak sowie die Fragmentierung unter Abspaltung der $\mathrm{Sit} \mathrm{BuPh}_{2}$ und der $\mathrm{CH}\left(\mathrm{SiMe}_{3}\right)_{2}$-Substituenten beobachtet.

Überraschend führt die Reaktion von $t \mathrm{BuPh}_{2} \mathrm{SiPLi}_{2}$ mit $\mathrm{R} * \mathrm{SbCl}_{2}\left(\mathrm{R}^{*}=\mathrm{CH}\left(\mathrm{SiMe}_{3}\right)_{2}\right)$ nicht zu einer Ringverbindungen analog $\mathrm{zu} \mathbf{1}$, stattdessen wird die gelbe Verbindung $\left[t \mathrm{BuPh}_{2} \mathrm{SiP}\left\{\mathrm{SbClCH}\left(\mathrm{SiMe}_{3}\right)_{2}\right\}_{2}\right]$ (2) erhalten. Verbindung 2 kristallisiert in der Raumgruppe

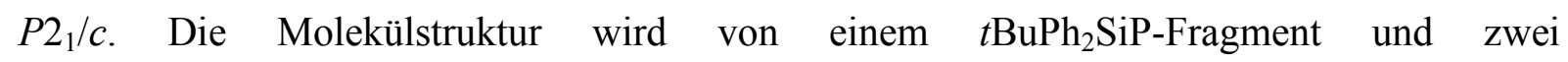
$\mathrm{Sb}(\mathrm{Cl}) \mathrm{CH}\left(\mathrm{SiMe}_{3}\right)_{2}$-Gruppen aufgebaut. Eines der Cl-Atome koordiniert an das benachbarte Metallatom und befindet sich so in einer stark asymmetrischen Brückenposition. Das Verhältnis von der langen zu der kurzen $\mathrm{Sb}-\mathrm{Cl}$-Bindung innerhalb dieser Brücken beträgt 1.39. Der zweite Chloroligand zeigt keine sekundären Wechselwirkungen, weder intra- noch intermolekular. Das $\mathrm{Sb}_{2} \mathrm{P}$-Fragment in $\mathbf{2}$ bildet einen Winkel von $99.4^{\circ}$, die siliziumorganischen Substituenten an den Antimonatomen zeigen eine cis-Orientierung zueinander, der $\mathrm{Si}_{\mathrm{BuPh}}$-Substituent am Phosphoratom steht dazu trans. Die Sb-PBindungen in 2 liegen mit Werten von 254.4 und $255.3 \mathrm{pm}$ im üblichen Bereich für Einfachbindungen zwischen diesen Elementen [6, 7]. Das ${ }^{31} \mathrm{P}\left\{{ }^{1} \mathrm{H}\right\}$-NMR-Spektrum von 2 zeigt ein Singulett bei $-166.8 \mathrm{ppm}, \mathrm{im}{ }^{29} \mathrm{Si}\left\{{ }^{1} \mathrm{H}\right\}$-NMR werden zwei Dublettsignale für die $\mathrm{SiMe}_{3}$-Gruppen bei 1,6 und 2,8 ppm beobachtet, sowie ein weiteres Dublett des $\mathrm{Sit} \mathrm{BuPh}_{2}-$ Rests bei 7,9 ppm. 


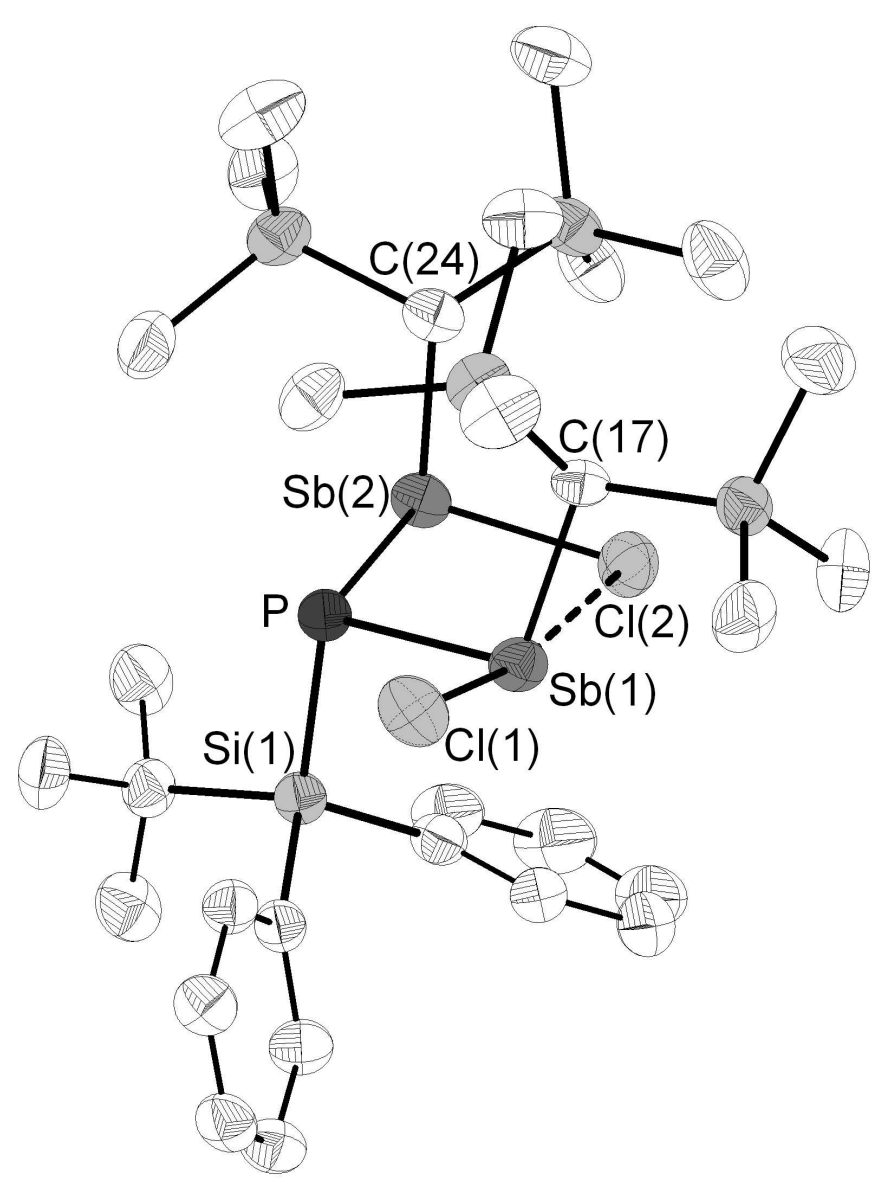

Abbildung 2 Molekülstruktur von 2 im Kristall (40\% Wahrscheinlichkeit für die Schwingungsellipsoide, die Wasserstoffatome sind nicht gezeigt).

Ausgesuchte Bindungslängen/pm und -winkel/ ${ }^{\circ}: \mathrm{Sb}(1)-\mathrm{P} 254.5(2), \mathrm{Sb}(2)-\mathrm{P} 255.3(2), \mathrm{P}-\mathrm{Si}(1)$ 229.6(2), Sb(1)-Cl(1) 240.1(2), Sb(2)-Cl(2) 239.4(2), Sb(1)-Cl(2) 331.7(2), Sb(1)-C(17) 218.6(6), Sb(2)-C(24) 216.9(6); $\mathrm{Si}(1)-\mathrm{P}-\mathrm{Sb}(1)$ 99.69(7), Si(1)-P-Sb(2) 95.78(7), Sb(1)-P$\mathrm{Sb}(2)$ 99.39(6), $\mathrm{P}-\mathrm{Sb}(1)-\mathrm{Cl}(1)$ 95.57(7), $\mathrm{P}-\mathrm{Sb}(2)-\mathrm{Cl}(2)$ 96.89(6).

Die zu 2 analoge Bismutverbindung $\left[t \mathrm{BuPh}_{2} \mathrm{SiP}\left\{\mathrm{BiClCH}\left(\mathrm{SiMe}_{3}\right)_{2}\right\}_{2}\right]$ (3) kann durch Umsetzung von $t \mathrm{BuPh}_{2} \mathrm{SiPLi}_{2}$ mit $\mathrm{R}^{*} \mathrm{BiCl}_{2}$ in Diethylether erhalten werden, sofern bei dieser Reaktion die Temperatur des Reaktionsgemischs nicht auf Werte oberhalb von $-20^{\circ} \mathrm{C}$ steigt. Verbindung 3 kristallisiert isotyp zu 2 und weist bezüglich der Bindungslängen innerhalb der Cl-Brücke ein Verhältnis von 1.25 auf. Die Bi-P-Bi-Gruppe bildet einen Winkel von 96.7, die Bi-P-Bindungen in 3 liegen mit Werten von 262.6 und $265.1 \mathrm{pm}$ im üblichen Bereich für Einfachbindungen zwischen diesen Elementen. So betragen die Bi-P-Bindungslängen beispielsweise in dem oben genannten phosphorsubstituierten Dibismuten $\left[\mathrm{BiP}\left(\mathrm{Si} t \mathrm{BuPh}_{2}\right)_{2}\right]_{2}$ 264.8 pm [7]. Die kristallene Verbindung 3 ist unter Luftausschluss über längere Zeit stabil, in 
Lösung zersetzt sich 3 hingegen schnell unter Bildung der Verbindungen $\mathbf{4}$ und $\mathbf{5}$, elementarem Bismut und weiterer Abbauprodukte.

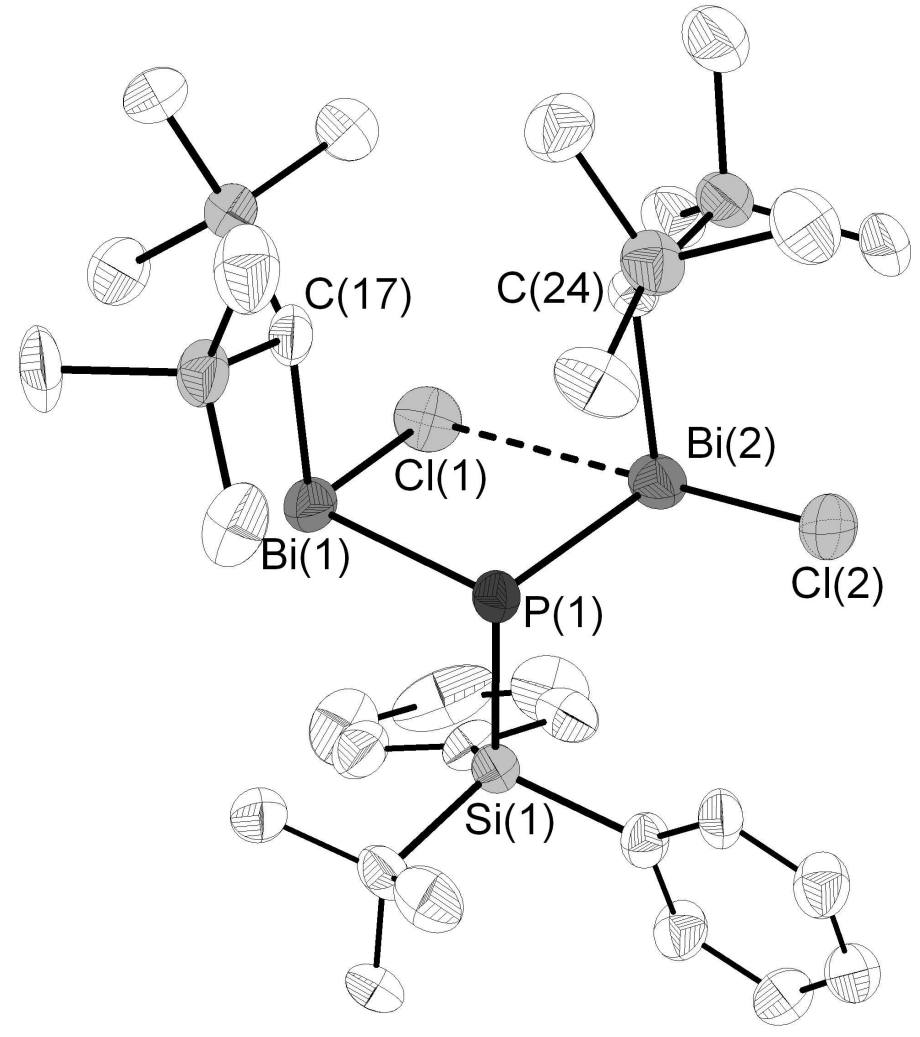

Abbildung 3 Molekülstruktur von 3 im Kristall (40\% Wahrscheinlichkeit für die Schwingungsellipsoide, die Wasserstoffatome sind nicht gezeigt).

Ausgesuchte Bindungslängen/pm und -winkel/ ${ }^{\circ}$ : Bi(1)-P 262.6(2), Bi(2)-P 265.1(2), P-Si(1) 228.6(3), $\mathrm{Bi}(1)-\mathrm{Cl}(1)$ 252.4(2), $\mathrm{Bi}(2)-\mathrm{Cl}(2)$ 253.3(2), $\mathrm{Bi}(1)-\mathrm{Cl}(2)$ 316.5(5), $\mathrm{Bi}(1)-\mathrm{C}(17)$ 228.8(8), Bi(2)-C(24) 228.7(7); Si(1)-P-Bi(1) 95.86(8), Si(1)-P-Bi(2) 98.48(8), Bi(1)-P$\mathrm{Bi}(2)$ 96.66(6), $\mathrm{P}-\mathrm{Bi}(1)-\mathrm{Cl}(1)$ 94.03(7), $\mathrm{P}-\mathrm{Bi}(2)-\mathrm{Cl}(2)$ 95.00(8).

Werden an Stelle von $t \mathrm{BuPh}_{2} \mathrm{SiPLi}_{2}$ die Dilithiumphosphandiide ThexMe $\mathrm{SiPLi}_{2}$ oder $\mathrm{Me}_{3} \mathrm{SiPLi}_{2}$ mit $\mathrm{R} * \mathrm{BiCl}_{2}$ in Ether bei $-50^{\circ} \mathrm{C}$ umgesetzt und die Reaktionsgemische anschließend auf Raumtemperatur erwärmt so bilden sich dunkle Lösungen, aus denen rote Kristalle der Verbindungen $\left[\mathrm{P}\left\{\mathrm{BiClCH}\left(\mathrm{SiMe}_{3}\right)_{2}\right\}_{3}\right]$ (4) bzw. $\left[\mathrm{P}_{2}\left\{\mathrm{BiClCH}\left(\mathrm{SiMe}_{3}\right)_{2}\right\}_{4}\right]$ (5) isoliert werden können. Die beiden Verbindungen 4 und 5 sind anders als die Verbindungen $\mathbf{1}$ bis 3 in gängigen organischen Lösungsmitteln weitgehend unlöslich und neigen bei Erwärmung zur Zersetzung unter Abscheidung von elementarem Bismut. Eine NMRspektroskopische Untersuchung dieser beiden Verbindungen in Lösung war infolgedessen nicht möglich. 
Bei der Synthese von Verbindung 4 ist es zur vollständigen Substitution sowohl der Lithiumkationen als auch des $\mathrm{SiMe}_{2}$ Thex-Restes durch $\mathrm{BiR}^{*} \mathrm{Cl}-$ Gruppen gekommen. Das $\mathrm{PBi}_{3}$-Fragment weist eine für tertiäre Phosphane typische pyramidale Struktur auf, die Bi-PBi-Winkel betragen $97.0^{\circ}$. Wie in Verbindung 3 weisen die Cl-Substituenten eine asymmetrisch verbrückende Position zwischen den Bi-Atomen auf. Das Verhältnis von langer zu kurzer Bi-Cl-Bindung beträgt in dieser Verbindung 1.21. Zusätzlich bilden sich im Kristall schwache intermolekulare Bi-Cl-Bindungen mit einer Länge von 349.1 pm aus, indem sich benachbarte $\mathrm{BiCl}$-Gruppen unter Ausbildung von rautenförmigen $\mathrm{Bi}_{2} \mathrm{Cl}_{2}$-Ringen einander annähern. Dies führt zu einer Vernetzung der Moleküle senkrecht zur kristallographischen cAchse (siehe Abb. 5). Aufgrund dieser Vernetzung und der damit verbundenen höheren Koordinationszahlen an den $\mathrm{Bi}$ - und $\mathrm{Cl}$-Atomen sind die kurzen Bi-Cl-Bindungen in $\mathbf{4}$ mit 263.0 pm um ca. 10 pm länger als die entsprechenden Bindungen in Verbindung 3, bei der keine intermolekularen Wechselwirkungen im Kristall beobachtet werden. Ähnliche interund intramolekulare Bi-Cl-Bindungslängen werden auch in metallorganisch substituierten Bismutchloriden beobachtet: So sind diese Bindungen beispielsweise in der Verbindung $\left[\mathrm{C}_{5} \mathrm{H}_{3} t \mathrm{Bu}_{2}(\mathrm{CO})_{2} \mathrm{FeBiCl}_{2}\right.$ ] zwischen 260.5 und 324.5 pm lang [8]. 


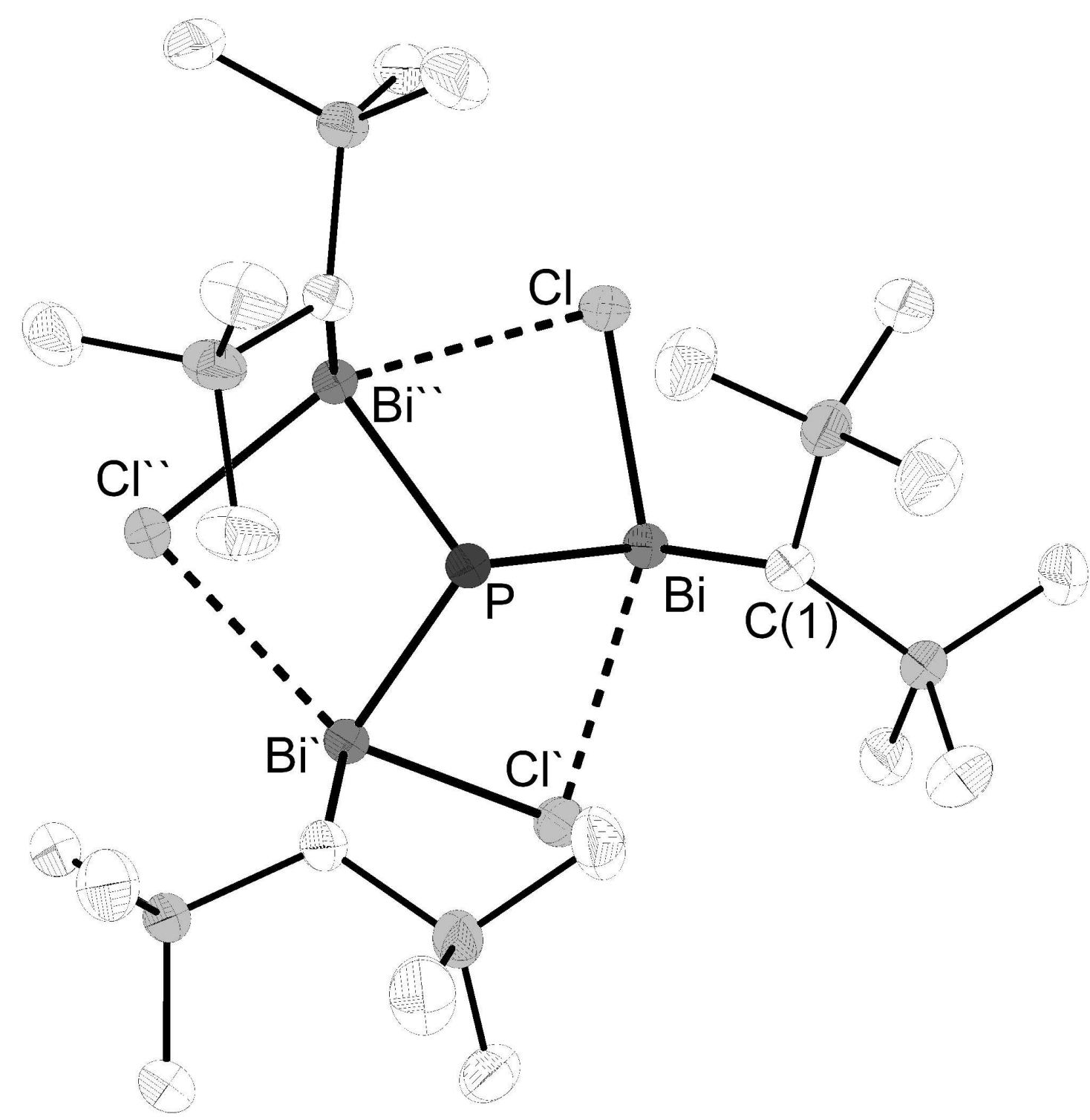

Abbildung 4 Molekülstruktur von 4 im Kristall (50\% Wahrscheinlichkeit für die Schwingungsellipsoide, die Wasserstoffatome sind nicht gezeigt).

Ausgesuchte Bindungslängen $/ \mathrm{pm}$ und -winkel/ ${ }^{\circ}$ : Bi-P 263.2(1), Bi-Cl 263.0(1), Bi-Cl 317.0(1), Bi-C(1) 228.6(4); Bi-P-Bi` 97.00(5), Bi-Cl-Bi“ 85.09(8). 


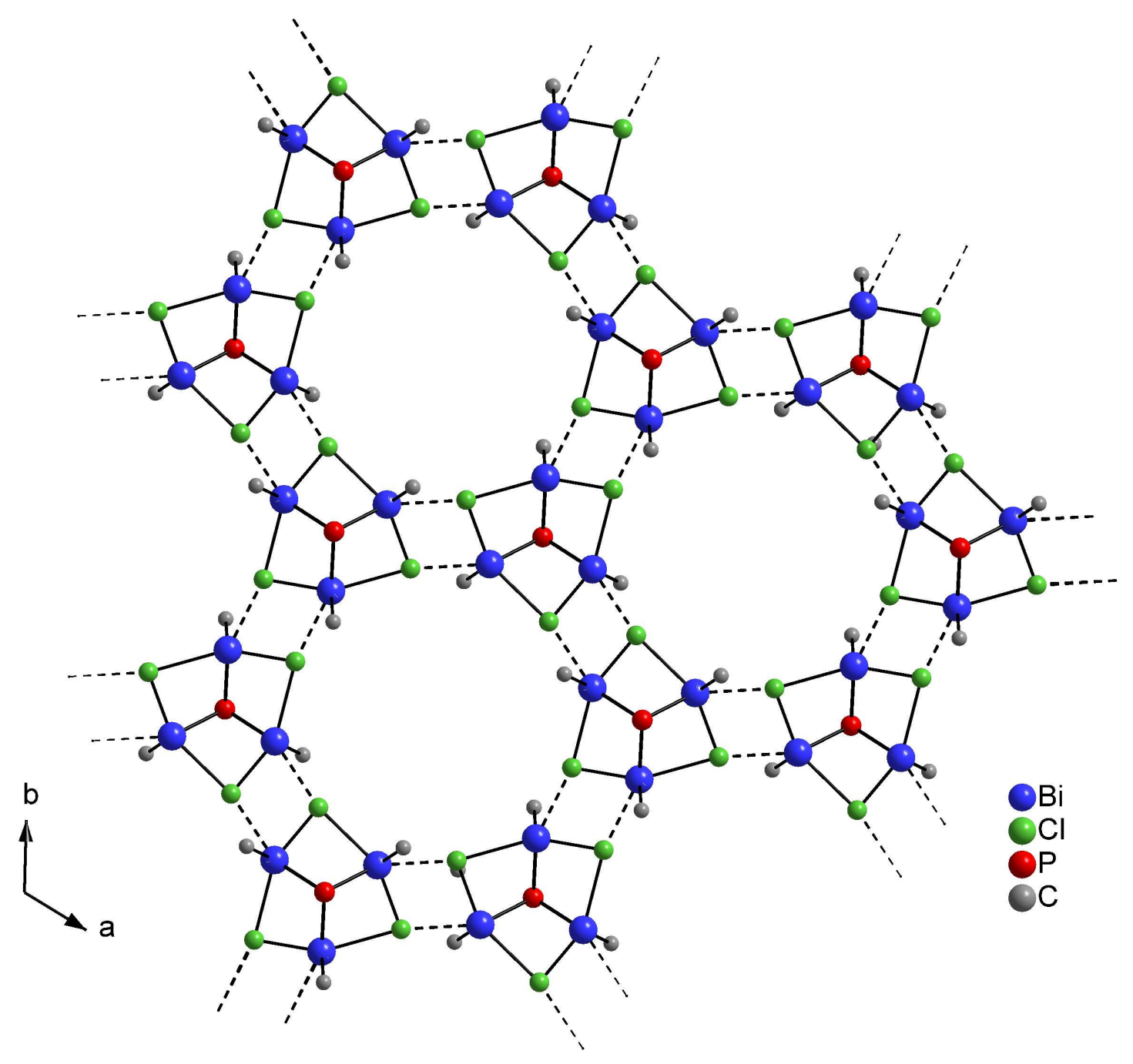

ODER 


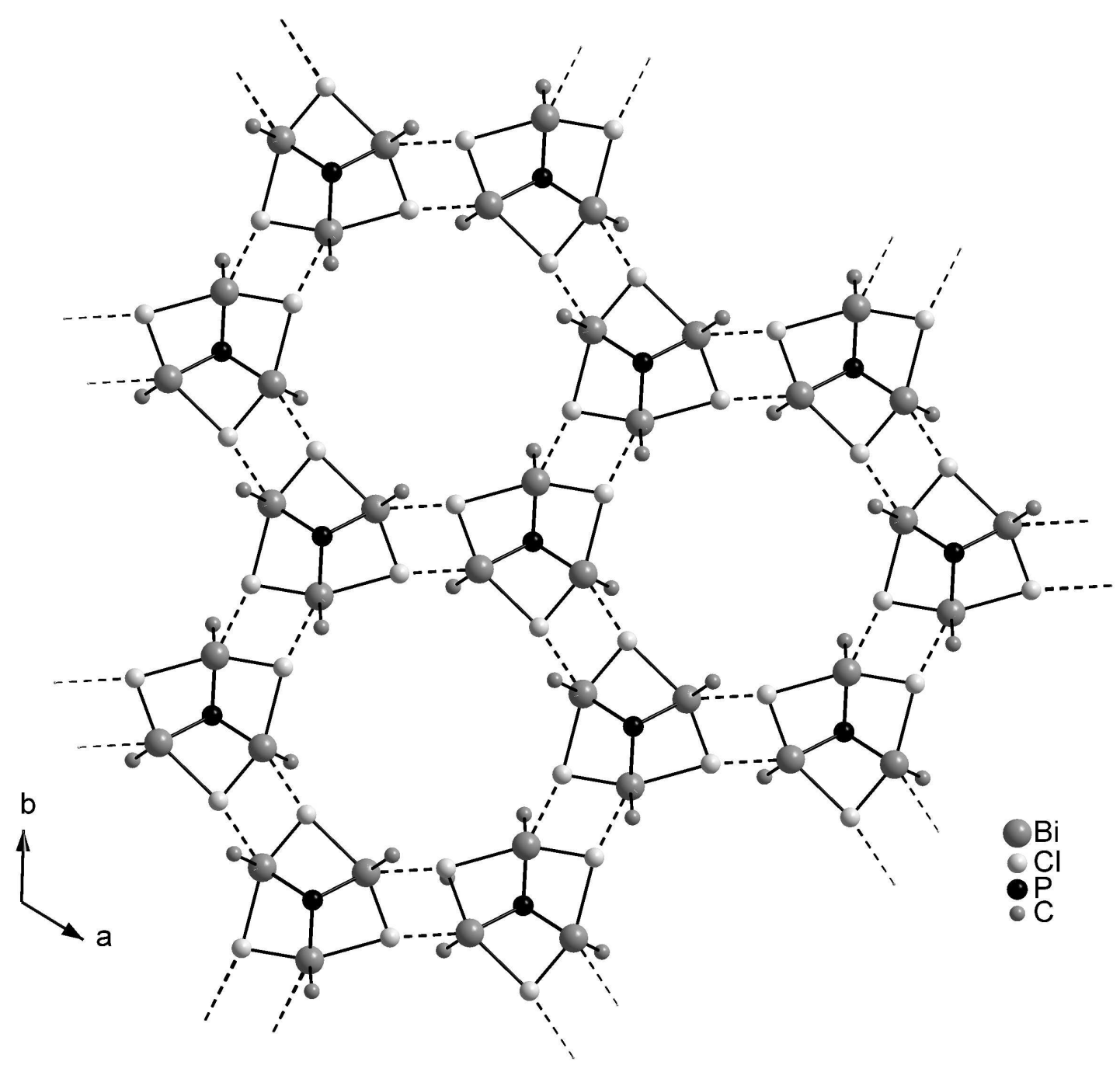

Abbildung 5 Netzstruktur von Verbindung 4 durch intermolekulare Bi-ClWechselwirkungen. Von den $\mathrm{CH}\left(\mathrm{SiMe}_{3}\right)_{2}$-Substituenten sind nur die $\alpha$-Kohlenstoffatome abgebildet.

Die Kristallstrukturanalyse von Verbindung 5 erfolgt in der triklinen Raumgruppe $P-1$ und zeigt, dass es zur Abspaltung der Silylgruppen am Phosphor und zur Knüpfung einer P-PBindung gekommen ist. Die entstandene $\mathrm{P}_{2}$-Einheit bindet an vier Bi-Atome, die ihrerseits an je einen $\mathrm{CH}\left(\mathrm{SiMe}_{3}\right)_{2}$-Rest gebunden sind und durch verbrückende Chloroliganden miteinander verknüpft sind. Damit hat das Molekül die Gestalt einer von einem $\mathrm{Bi}_{4} \mathrm{Cl}_{4}$-Ring umgebenen $\mathrm{P}_{2}$-Einheit. Anders als in den Bismutverbindungen $\mathbf{3}$ und $\mathbf{4}$ sind die Cl-Brücken in 5 weitgehend symmetrisch: Die Bi-Cl-Bindungslängen liegen alle im Bereich von 268.0 bis 287.6 pm. Der $\mathrm{Bi}_{4} \mathrm{Cl}_{4}$-Ring ist praktisch planar, die $\mathrm{P}_{2}$-Einheit liegt hingegen nicht in dieser Ebene, sondern ist aus dieser um einen Winkel von ca. $54^{\circ}$ herausgedreht (siehe Abbildung 
7). Infolge dieser Verkippung der $\mathrm{P}_{2}$-Einheit gegenüber dem Ring betragen an den Phosphoratomen alle Winkel nahezu 90 .

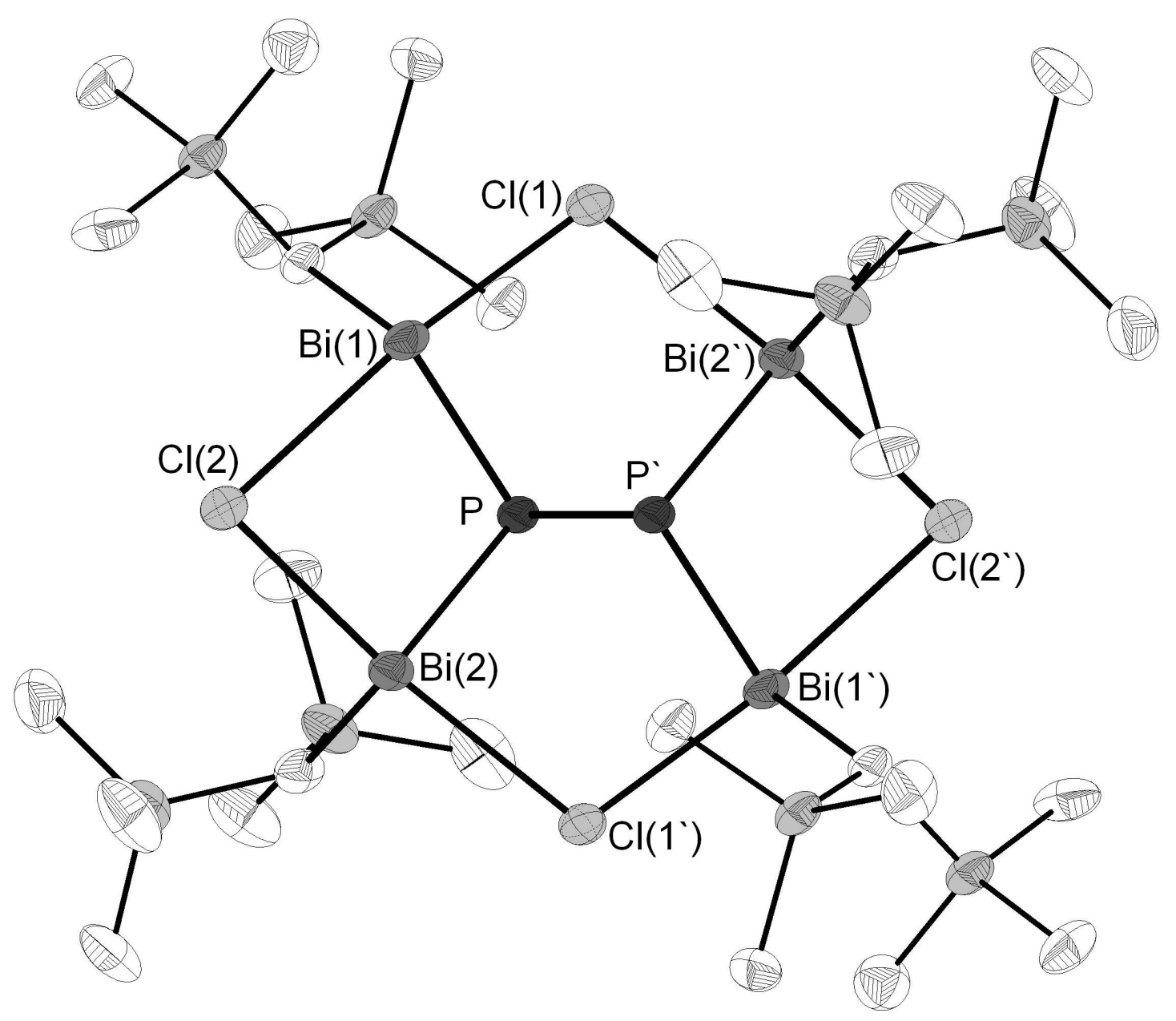

Abbildung 6 Molekülstruktur von 5 im Kristall (40\% Wahrscheinlichkeit für die Schwingungsellipsoide, die Wasserstoffatome sind nicht gezeigt).

Ausgesuchte Bindungslängen/pm und -winkel/ ${ }^{\circ}$ : Bi(1)-P 265.9(3), Bi(2)-P 265.2(3), P-P` 222.6(7), $\mathrm{Bi}(1)-\mathrm{Cl}(1)$ 268.0(4), $\mathrm{Bi}(1)-\mathrm{Cl}(2)$ 287.6(4), $\mathrm{Bi}(2)-\mathrm{Cl}(2)$ 266.9(3), $\mathrm{Bi}(2)-\mathrm{Cl}\left(1^{\prime}\right)$ 285.3(4); P`-P-Bi(1) 92.1(1), P`-P-Bi(2) 92.8(2), Bi(1)-P-Bi(2) 92.3(1), Bi(1)-Cl(1)-Bi(2`) 105.8(1), $\quad \mathrm{Bi}(1)-\mathrm{Cl}(2)-\mathrm{Bi}(2) \quad 86.7(1), \quad \mathrm{Cl}(1)-\mathrm{Bi}(1)-\mathrm{Cl}(2) \quad$ 172.1(1), $\quad \mathrm{Cl}\left(1^{\prime}\right)-\mathrm{Bi}(2)-\mathrm{Cl}(2)$ 173.6(1). 


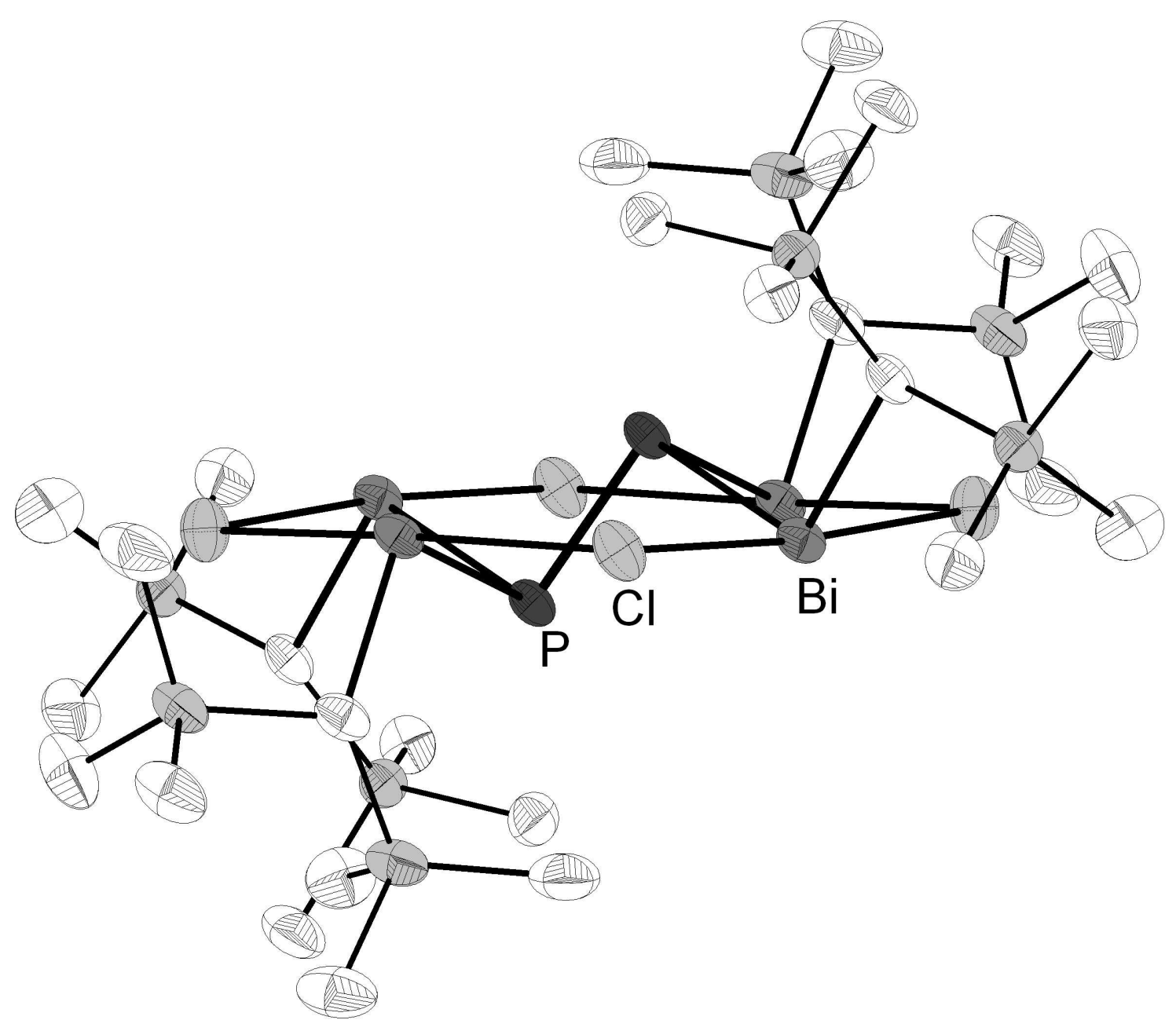

Abbildung 7 Molekülstruktur von 5 im Kristall (Seitenansicht)

\section{Zusammenfassung und Ausblick}

Die Reaktion des Dilithiumphosphandiids $t \mathrm{BuPh}_{2} \mathrm{SiPLi}_{2}$ mit dem Arsenchlorid $\mathrm{R}^{*} \mathrm{AsCl}_{2}\left(\mathrm{R}^{*}\right.$ $\left.=\mathrm{CH}\left(\mathrm{SiMe}_{3}\right)_{2}\right)$ führt $\mathrm{zu}$ der cyclischen Verbindung 1 mit einem zentralen $\mathrm{As}_{2} \mathrm{P}_{2}$-Ring. Hingegen führen Reaktionen desselben Phosphandiids mit den analogen Chloriden der schwereren Homologen ( $\mathrm{Sb}$ und $\mathrm{Bi}$ ) - auch bei einer Durchführung in einem 1:1-Verhähtnis der Ausgangsverbindungen - zu den chloroverbrückten Spezies 2 und 3. Dies kann auf die stabilisierende Wirkung der Chlorobrücken zurückgeführt werden, die bei Arsen nicht in gleichem Maße zu erwarten ist. Werden Dilithiumsilylphosphandiide mit sterisch weniger anspruchsvollen Silylsubstituenten mit $\mathrm{R} * \mathrm{BiCl}_{2}$ umgesetzt, so beobachtet man die Abspaltung der Silylreste, die vermutlich zu den entsprechenden Chlorsilanen umgesetzt werden. Im Fall des ThexMe $\mathrm{S}_{2} \mathrm{Si}$-substituierten Phosphandiids führt dies zur Ausbildung eines 
Tribismutylphosphans (Verbindung 4), während bei Verwendung des $\mathrm{Me}_{3} \mathrm{Si}$-Substituenten, in folge von Redoxprozessen ein entsprechend substituiertes Diphosphan entsteht (Verbindung 5) Diese oxidative Phosphor-Phosphor-Bindungsknüpfung geht einher mit der Bildung von elementarem Bismut, so das die Vermutung nahe liegt, dass das eingesetzte $\mathrm{R}^{*} \mathrm{BiCl}_{2}$ hier als oxidationsmittel wirkt.

Insbesondere die ungewöhnlichen Verbindungen $\mathbf{4}$ und 5, die als die ersten zwei Glieder einer homologen Reihe von vollständig bismutylsubstituierten Phosphanen aufgefasst werden können, zeigen, dass auf diesem noch weitgehend unerforschten Gebiet der molekularen binären Verbindungen der Gruppe-15-Elemente noch so manche Überraschung zu erwarten ist. Gegenstand laufender Untersuchungen ist die Synthese weiterer Molekülverbindungen mit $\mathrm{P}-\mathrm{Sb}$ - und P-Bi-Bindungen sowie von Verbindungen der Elementkombinationen As/Sb und $\mathrm{As} / \mathrm{Bi}$.

\section{Experimentelles}

Alle Arbeiten wurden unter Ausschluss von Luft und Feuchtigkeit in einer Atmosphäre von gereinigtem Stickstoff ausgeführt. Die verwendeten Lösungsmittel wurden getrocknet und vor Benutzung frisch destilliert. Die NMR-Spektren wurden an einem Gerät vom Typ DPX Avance 300 der Firma Bruker aufgenommen. $t \mathrm{BuPh}_{2} \mathrm{SiPH}_{2}$, ThexMe $\mathrm{SiPH}_{2}$ und $\mathrm{Me}_{3} \mathrm{SiPH}_{2}$ sowie die Verbindungen $\left(\mathrm{Me}_{3} \mathrm{Si}\right)_{2} \mathrm{CHMCl}_{2}(\mathrm{M}=\mathrm{As}, \mathrm{Sb}, \mathrm{Bi})$ wurden nach bekannten Literaturvorschriften synthetisiert $[7 \mathrm{~b}, 10]$.

1: $1.04 \mathrm{~g}$ (3.82 mmol) $t \mathrm{BuPh}_{2} \mathrm{SiPH}_{2}$ werden in $30 \mathrm{ml}$ Diethylether gelöst und auf $0^{\circ} \mathrm{C}$ gekühlt. $\mathrm{Zu}$ dieser Lösung werden langsam $4.8 \mathrm{ml}$ 1.6 M (7.64 mmol) $n$ BuLi-Lösung zugetropft. Nach beendeter Zugabe wird die Lösung auf Raumtemperatur erwärmt und weitere 30 Minuten gerührt. Daraufhin wird das Reaktionsgemisch auf $-30{ }^{\circ} \mathrm{C}$ gekühlt und eine Lösung von 1.17 g (3.82 mmol) $\mathrm{R}^{*} \mathrm{AsCl}_{2}\left(\mathrm{R}^{*}=\mathrm{CH}\left(\mathrm{SiMe}_{3}\right)_{2}\right)$ gelöst in $10 \mathrm{ml}$ Diethylether zugegeben. Anschließend wird das Reaktionsgemisch auf Raumtemperatur erwärmt und weitere 16 Stunden gerührt. Nach dem Entfernen des Lösungsmittels wird der verbliebene gelbe Feststoff in Pentan aufgenommen. Nach Filtration fallen aus dieser Lösung bei $-30{ }^{\circ} \mathrm{C}$ gelbe Kristalle der Verbindung 1 aus. Ausbeute: 2.60 g (68\%). Elementaranalyse (\%) berechnet für: $\mathrm{C}_{46} \mathrm{H}_{76} \mathrm{As}_{2} \mathrm{P}_{2} \mathrm{Si}_{6}$ (1009.40): C 54.73, H 7.59; gefunden: C 54,48 H 7.22. 
${ }^{1} \mathbf{H}-\mathbf{N M R}\left(\mathrm{C}_{6} \mathrm{D}_{6}, 25{ }^{\circ} \mathrm{C}\right): \delta=-0.02\left(\mathrm{t},{ }^{3} \mathrm{~J}_{\mathrm{P}, \mathrm{H}}=7.9 \mathrm{~Hz}, \mathrm{CH}\left(\mathrm{SiMe}_{3}\right)_{2}, 2 \mathrm{H}\right), 0.41\left(\mathrm{~s},\left(\mathrm{SiCH}_{3}, 36 \mathrm{H}\right)\right.$, $1.30(\mathrm{~s}, t \mathrm{Bu}, 18 \mathrm{H}), 7.29(\mathrm{~m}, \mathrm{Ph}, 12 \mathrm{H}), 7.91(\mathrm{~m}, \mathrm{Ph}, 8 \mathrm{H}) ;{ }^{31} \mathbf{P}\left({ }^{1} \mathbf{H}\right)-\mathbf{N M R}\left(\mathrm{C}_{6} \mathrm{D}_{6}\right): \delta=-97.7(\mathrm{t}$, $\left.{ }^{3} \mathrm{~J}_{\mathrm{P}, \mathrm{H}}=7.9 \mathrm{~Hz}\right) ; \mathbf{M S}\left(\mathrm{EI}, 70 \mathrm{eV}, 160{ }^{\circ} \mathrm{C}\right) \mathrm{m} / \mathrm{z}(\%): 1008(55)\left[\mathrm{M}^{+}\right], 849$ (100) $\left[\mathrm{M}^{+}{ }_{-}\right.$ $\left.\mathrm{CH}\left(\mathrm{SiMe}_{3}\right)_{2}\right], 769$ (10) $\left[\mathrm{M}^{+}-t \mathrm{BuPh}_{2} \mathrm{Si}\right], 595$ (100) $\left[\mathrm{M}^{+}-\mathrm{CH}\left(\mathrm{SiMe}_{3}\right)_{2},-t \mathrm{BuPh}_{2} \mathrm{Si},-\mathrm{Me}\right], 540$ (45) $\left(\mathrm{M}^{+} / 2\right), 239(100)\left[t \mathrm{BuPh}_{2} \mathrm{Si}^{+}\right], 73(100)\left[\mathrm{Me}_{3} \mathrm{Si}+\right]$. IR (KBr): 3070(w), 3049(w), 2948(s), 2893(m), 2856(m), 1953(vw), 1897(vw), 1818(vw)1652(w), 1587(w), 1464(m), 1427(m), 1391(w), 1362(w), 1300(vw), 1248(s), 1190(w), 1157(vw), 1101(s), 998(m), 939(vw), 842(vs), 764(m), 736(m), 699(s), 665(m) 603(m), 510(s) 484(s), 404(w).

2: $0.11 \mathrm{~g}(0.41 \mathrm{mmol}) t \mathrm{BuPh}_{2} \mathrm{SiPH}_{2}$ werden in $5 \mathrm{ml}$ Diethylether gelöst und auf $0^{\circ} \mathrm{C}$ gekühlt. $\mathrm{Zu}$ dieser Lösung werden langsam $0.51 \mathrm{ml} 1.6 \mathrm{M}(0.82 \mathrm{mmol}) n$ BuLi-Lösung zugetropft. Nach beendeter Zugabe wird die Lösung auf Raumtemperatur erwärmt und weitere 30 Minuten gerührt. Daraufhin wird das Reaktionsgemisch auf $-50{ }^{\circ} \mathrm{C}$ gekühlt und eine Lösung von $0.29 \mathrm{~g}(0.82 \mathrm{mmol}) \mathrm{R} * \mathrm{SbCl}_{2}\left(\mathrm{R}^{*}=\mathrm{CH}\left(\mathrm{SiMe}_{3}\right)_{2}\right)$ gelöst in $5 \mathrm{ml}$ Diethylether zugegeben. Daraufhin wird das Reaktionsgemisch auf Raumtemperatur erwärmt, weitere zwei Stunden gerührt und das entstandene Lithiumchlorid durch Filtration entfernt. Aus der so erhaltenen klaren gelben Lösung fallen bei $5{ }^{\circ} \mathrm{C}$ gelbe Kristalle der Verbindung 2 aus. Ausbeute: $0.2 \mathrm{~g}$ (54\% bezogen auf Antimon). Elementaranalyse (\%) berechnet für: $\mathrm{C}_{30} \mathrm{H}_{57} \mathrm{Cl}_{2} \mathrm{PSb}_{2} \mathrm{Si}_{5}$ (903.6): C 39.88, H 6.36; gefunden: C 39.42, H 6.74.

${ }^{1}$ H-NMR (Tol.-D $\left.8,25{ }^{\circ} \mathrm{C}\right): \delta=0.22\left(\mathrm{~s},\left(\mathrm{SiCH}_{3}, 18 \mathrm{H}\right), 0.46\left(\mathrm{~s},\left(\mathrm{SiCH}_{3}, 18 \mathrm{H}\right), 1.34(\mathrm{~s}, t \mathrm{Bu}\right.\right.$, 9H), $1.48\left(\mathrm{~d},{ }^{3} \mathrm{~J}_{\mathrm{P}, \mathrm{H}}=2.7 \mathrm{~Hz}, \mathrm{CH}\left(\mathrm{SiMe}_{3}\right)_{2}, 2 \mathrm{H}\right), 7.29$ (m, Ph, 6H), 7.91 (m, Ph, 4H); ${ }^{31} \mathbf{P}-\mathbf{N M R}$ (Tol.-D $8,25^{\circ} \mathrm{C}$ ): $\delta=-165.3$ (s); IR (KBr): 3071(w), 3049(w), 2949(s), 2891(m), 2855(m), 1485(w), 1468(w), 1459(w), 1427(m), 1360(w), 1251(vs), 1192(w), 1100(m), 1007(w), 990(m), 970(m), 939(w), 837(vs), 764(m), 738(w), 697(s), 661(m), 599(m), 518(w), 509(s), 483(m), 448(w), 408(w).

3: $0.16 \mathrm{~g}$ (0.59 mmol) $t \mathrm{BuPh}_{2} \mathrm{SiPH}_{2}$ werden in $10 \mathrm{ml}$ Diethylether gelöst und auf $-20{ }^{\circ} \mathrm{C}$ gekühlt. $\mathrm{Zu}$ dieser Lösung werden langsam $0.75 \mathrm{ml} 1.6 \mathrm{M}$ (1.2 mmol) nBuLi-Lösung zugetropft. Nach beendeter Zugabe wird die Lösung auf Raumtemperatur erwärmt und weitere 30 Minuten gerührt. Daraufhin wird das Reaktionsgemisch auf $-60{ }^{\circ} \mathrm{C}$ gekühlt und eine Lösung von $0.52 \mathrm{~g}(0.59 \mathrm{mmol}) \mathrm{R}^{*} \mathrm{BiCl}_{2}\left(\mathrm{R}^{*}=\mathrm{CH}\left(\mathrm{SiMe}_{3}\right)_{2}\right)$ gelöst in $10 \mathrm{ml}$ Diethylether zugegeben. Anschließend wird das Reaktionsgemisch langsam bis auf $-20{ }^{\circ} \mathrm{C}$ erwärmt und bei dieser Temperatur zentrifugiert. Die so erhaltene orange Lösung wird bis auf ein Volumen von ca. $3 \mathrm{ml}$ reduziert und auf $-30{ }^{\circ} \mathrm{C}$ gekühlt. Innerhalb von zwei Tagen bilden 
sich gelbe Kristalle der Verbindung 3. Ausbeute: $0.19 \mathrm{~g}$ (60\% bezogen auf Bismut). Elementaranalyse (\%) berechnet für: $\mathrm{C}_{30} \mathrm{H}_{57} \mathrm{Bi}_{2} \mathrm{Cl}_{2} \mathrm{PSi}_{5}$ (1078.0): C 33.42, $\mathrm{H}$ 5.33; gefunden: C 32.84, H 5.10.

${ }^{1}$ H-NMR (Tol.-D $\left.8,25^{\circ} \mathrm{C}\right): \delta=0.21\left(\mathrm{~s},\left(\mathrm{SiCH}_{3}, 18 \mathrm{H}\right), 0.51\right.$ (s, $\left(\mathrm{SiCH}_{3}, 18 \mathrm{H}\right), 1.35(\mathrm{~s}, t \mathrm{Bu}$, 9H), 1.62 (s, br, $\left.\mathrm{CH}\left(\mathrm{SiMe}_{3}\right)_{2}, 2 \mathrm{H}\right), 7.25$ (m, Ph, 6H), 7.84 (m, Ph, 4H); ${ }^{31}$ P-NMR (Tol.-D $\left.25^{\circ} \mathrm{C}\right): \delta=-158.3(\mathrm{~s})$; IR (KBr): 3069(w), 3049(w), 2947(vs), 2892(s), 2856(m), 1484(w), 1468(m), 1427(s), 1356(w), 1360(m), 1250(vs), 1191(w), 1100(m), 1009(s), 997(s), 943(m), 907(w), 839(vs), 772(m), 737(m), 699(s), 657(s), 596(m), 512(s), 483(s), 449(w), 408(w).

4: $0.10 \mathrm{~g}$ (0.57 mmol) ThexMe $\mathrm{SiPH}_{2}$ werden in $10 \mathrm{ml}$ Diethylether gelöst und auf $0{ }^{\circ} \mathrm{C}$ gekühlt. $\mathrm{Zu}$ dieser Lösung werden langsam $0.71 \mathrm{ml} 1.6 \mathrm{M}$ (1.14 mmol) $n$ BuLi-Lösung zugetropft. Nach beendeter Zugabe wird die Lösung auf Raumtemperatur erwärmt und weitere 30 Minuten gerührt. Daraufhin wird das Reaktionsgemisch auf $-50{ }^{\circ} \mathrm{C}$ gekühlt und eine Lösung von $0.48 \mathrm{~g}(1.1 \mathrm{mmol}) \mathrm{R} * \mathrm{BiCl}_{2}\left(\mathrm{R}^{*}=\mathrm{CH}\left(\mathrm{SiMe}_{3}\right)_{2}\right)$ gelöst in $15 \mathrm{ml}$ Diethylether zugegeben. Anschließend wird das Reaktionsgemisch langsam auf Raumtemperatur erwärmt und nach 20 Stunden von dem entstandenen Niederschlag $(\mathrm{LiCl})$ befreit. Die so erhaltene rote Lösung wird bis auf ein Volumen von $10 \mathrm{ml}$ reduziert und auf $-30{ }^{\circ} \mathrm{C}$ gekühlt. Innerhalb von drei Tagen bilden sich rote sechseckige plättchenförmige Kristalle der Verbindung 4. Ausbeute: $0.12 \mathrm{~g}$ (26\% bezogen auf Bismut). Elementaranalyse (\%) berechnet für: $\mathrm{C}_{21} \mathrm{H}_{57} \mathrm{Bi}_{3} \mathrm{Cl}_{3} \mathrm{PSi}_{6}$ (1242.5): C $20.30 \mathrm{H}$ 4.62; gefunden: C 20.30, H 4.54.

IR (KBr): 2952(s), 2897(m), 1423(w), 1402(w), 1251(vs), 1099(s), 1011(s), 969(w), 942(w), 842(vs), 771(w), 756(w), 688(m), 658(s), 592(m), 463(w).

5: $0.04 \mathrm{~g}(0.38 \mathrm{mmol}) \mathrm{Me}_{3} \mathrm{SiPH}_{2}$ werden in $10 \mathrm{ml}$ Diethylether gelöst und auf $0{ }^{\circ} \mathrm{C}$ gekühlt. $\mathrm{Zu}$ dieser Lösung werden langsam $0.47 \mathrm{ml} 1.6 \mathrm{M}(0.76 \mathrm{mmol}) n$ BuLi-Lösung zugetropft. Nach beendeter Zugabe wird die Lösung auf Raumtemperatur erwärmt und weitere 30 Minuten gerührt. Daraufhin wird das Reaktionsgemisch auf $-50{ }^{\circ} \mathrm{C}$ gekühlt und eine Lösung von $0.45 \mathrm{~g}(1.0 \mathrm{mmol}) \mathrm{R}^{*} \mathrm{BiCl}_{2}\left(\mathrm{R}^{*}=\mathrm{CH}\left(\mathrm{SiMe}_{3}\right)_{2}\right)$ gelöst in $15 \mathrm{ml}$ Diethylether zugegeben. Anschließend wird das Reaktionsgemisch langsam auf Raumtemperatur erwärmt und nach 20 Stunden von dem entstandenen Niederschlag ( $\mathrm{LiCl}$ und $\mathrm{Bi}$ ) befreit. Die so erhaltene rote Lösung wird auf $-30{ }^{\circ} \mathrm{C}$ gekühlt. Innerhalb von drei Tagen bilden sich rote stäbchenförmige Kristalle der Verbindung 5 $\mathrm{Et}_{2} \mathrm{O}$. Ausbeute: $0.10 \mathrm{~g}$ (30\% bezogen auf Phosphor). Elementaranalyse (\%) berechnet für: $\mathrm{C}_{28} \mathrm{H}_{76} \mathrm{Bi}_{4} \mathrm{Cl}_{4} \mathrm{P}_{2} \mathrm{Si}_{8} \cdot \mathrm{C}_{4} \mathrm{H}_{10} \mathrm{O}$ (1751.4): C $21.95 \mathrm{H} 4.95$; gefunden: C 21.49, H 4.98 . 
IR (KBr): 2947(s), 2895(m), 2856(m), 1400(m), 1250(vs), 1191(w), 1099(w), 1009(m), 962(w), 941(w), 841(vs), 771(m), 686(w), 656(m), 590(w), 467(w).

Der Deutschen Forschungsgemeinschaft danken wir für die Förderung dieser Untersuchungen.

\section{Literatur}

[1] Übersichten: a) L. Balázs, H. J. Breunig, Coord. Chem. Rev. 2004, 248, 603-621; b) H. J. Breunig, R. Rösler, Coord. Chem. Rev. 1997, 163, 33-53; c) M. Baudler, Angew. Chem. 1987, 99, 429-451; Angew. Chem. Int. Ed. Engl. 1987, 26, 419-441; d) M. Baudler, Angew. Chem. 1982, 94, 520-539; Angew. Chem. Int. Ed. Engl. 1982, 21, 492512.

[2] a) J. Hahn, M. Baudler, Z. Naturforsch. 1982, 37b, 797-805; b) O. Mundt, G. Becker, H.-J. Wessely, Z. Anorg. Allg. Chem. 1982, 486, 70-89; c) M. Baudler, P. Bachmann, Angew. Chem. 1981, 93, 112-113; Angew. Chem. Int. Ed. Engl. 1981, 20, 123-124.

[3] a) M. Westerhausen, S. Weinrich, P. Mayer, Z. Anorg. Allg. Chem. 2003, 629, 11531156; b) G. Linti, W. Köstler, Z. Anorg. Allg. Chem. 2002, 628, 63-66; c) H. J. Breunig, R. Rösler, E. Lork, Angew. Chem. 1997, 109, 2333-2334; Angew. Chem. Int. Ed. Engl. 1997, 36, 2237-2238; d) C. von Hänisch, D. Fenske, Z. Anorg. Allg. Chem. 1997, 623, 1040-1042; e) M. Baudler, H. Jachow, B. Lieser, K.-F. Tebbe, M. Fehér, Angew. Chem. 1989, 101, 1245-1247; Angew. Chem. Int. Ed. Engl. 1989, 28, 1231-1232; f) C. Mujica, D. Weber, H.-G. Schnering, Z. Naturforsch. 1986, 41b, 991-999; g) M. Baudler, S. Wietfeldt-Haltenhoff, Angew. Chem. 1985, 97, 986-987; Angew. Chem. Int. Ed. Engl. 1985, 24, 991-992; h) M. Baudler, S. Wietfeldt-Haltenhoff, Angew. Chem. 1984, 96, 361-362; Angew. Chem. Int. Ed. Engl. 1984, 23, 379-380; i) M. Baudler, P. Bachmann, Z. Anorg. Allg. Chem. 1982, 485, 129-132; j) M. Baudler, J. Hellmann, P. Bachmann, K.-F. Tebbe, R. Fröhlich, M. Feher, Angew. Chem. 1981, 91, 415-417; Angew. Chem. Int. Ed. Engl. 1981, 20, 406-407; k) H.-G. von Schnering, D. Fenske, W. Hönle, M. Binnewies, K. Peters, Angew. Chem. 1979, 91, 755-756; Angew. Chem. Int. Ed. Engl. 1979, 18, 679; 1) A. Tzschach, V. Kiesel, J. praktische Chem. 1971, 313, 259-264. 
[4] E. L. Doyle, L. Riera, D. S. Wright, Eur. J. Inorg. Chem. 2003, 3279-3289.

[5] a) M. Baudler, S. Klautke, Z. Naturforsch. 1981, 36b, 527-531; b) M. Baudler, D. Habermann, Angew. Chem. 1979, 91, 939-940; Angew. Chem. Int. Ed. Engl. 1979, 18, $877-878$.

[6] a) A. Bashall, F. Garcia, G. T. Lawson, M. McPartlin, A. Rothenberger, A. D. Woods, D. S. Wright, Can. J. Chem. 2002, 80, 1421-1427; b) P. Jutzi, U. Meyer, S. Opiela, M. Olmstead, P. P. Power, Organometallics 1990, 9, 1459-1463; c) M. Baudler, Y. Aktalay, T. Heinlein, K.-F. Tebbe, Z. Naturforsch. 1982, 37b, 299-305.

[7] a) C. von Hänisch, D. Nikolova, Eur. J. Inorg. Chem. 2006, 4770-4773; b) C. von Hänisch, D. Nikolova, Eur. J. Inorg. Chem. 2005, 378-382.

[8] a) T. Gröer, M. Scheer, J. Chem. Soc. Dalton Trans. 2000, 647-653; b) W. Clegg, N. A. Compton, R. J. Errington, G. A. Fisher, D. C. R. Hockless, N. C. Norman, N. A. L. Williams, S. E. Statford, S. J. Nichols, P. S. Jarrett, A. G. Orpen, J. Chem. Soc. Dalton Trans. 1992, 193- 201; c) J. M. Wallis, G. Müller, H.Schmidbaur, J. Organomet. Chem. 1987, 325, 159-168.

[9] Die Datensammlungen wurden auf einem Flächendetektor STOE IPDS2 (Graphitmonochromator, Mo- $\mathrm{K}_{\alpha}-$ Strahlung) durchgeführt. Die Strukturlösungen erfolgten mit direkten Methoden, verfeinert gegen $F^{2}$ : $(\mathrm{Bi}, \mathrm{Sb}, \mathrm{As}, \mathrm{Cl}, \mathrm{P}, \mathrm{Si}, \mathrm{O}, \mathrm{C}$ anisotrop verfeinert, H-Atomlagen wurden berechnet. Verbindung 5 kristallisiert mit einem Molekül Diethylether, dieses wurde mit Splitlagen und ohne Wasserstoffatome verfeinert). Die kristallographischen Daten (ohne Strukturfaktoren) der in dieser Veröffentlichung beschriebenen Strukturen wurden als ,supplementary publication no. CCDC-724315 - 724319“ beim Cambridge Crystallographic Data Centre hinterlegt. Kopien der Daten können kostenlos bei folgender Adresse in Großbritannien angefordert werden: CCDC, 12 Union Road, Cambridge CB21EZ (Fax: (+44)1223-336033; E-mail: deposit@ccdc.cam.ac.uk).

[10] a) H. Althaus, H. J. Breunig, R. Rösler, E. Lork, Organometallics, 1999, 18, 328-331; b) G. Fritz, W. Schick, Z. Anorg. Allg. Chem. 1984, 511, 108-131; c) H. J. Breunig, W. Kanig, A. Soltani-Nesham, Polyhedron, 1983, 2, 291-292; d) M. J. S. Gynane, A. Hudson, M. F. Lappert, P. P. Power, H. Goldwhite, J. Chem. Soc. Dalton 1980, 24282433. 
Tabelle 1 Kristallographische Daten der Verbindungen 1-5 [9].

\begin{tabular}{|c|c|c|c|c|c|}
\hline Verbindung & 1 & 2 & 3 & 4 & 5 \\
\hline Formel & $\mathrm{C}_{46} \mathrm{H}_{76} \mathrm{As}_{2} \mathrm{P}_{2} \mathrm{Si}_{6}$ & $\mathrm{C}_{30} \mathrm{H}_{57} \mathrm{Cl}_{2} \mathrm{PSb}_{2} \mathrm{Si}_{5}$ & $\mathrm{C}_{30} \mathrm{H}_{57} \mathrm{Bi}_{2} \mathrm{Cl}_{2} \mathrm{PSi}_{5}$ & $\mathrm{C}_{21} \mathrm{H}_{57} \mathrm{Bi}_{3} \mathrm{Cl}_{3} \mathrm{PSi}_{6}$ & $\begin{array}{l}\mathrm{C}_{28} \mathrm{H}_{76} \mathrm{Bi}_{4} \mathrm{Cl}_{4} \mathrm{P}_{2} \mathrm{Si}_{8} \\
\mathrm{C}_{4} \mathrm{H}_{10} \mathrm{O}\end{array}$ \\
\hline Raumgruppe & $P 2_{1} / n$ & $P 2_{1} / c$ & $P 2_{1} / c$ & $R-3$ & $P-1$ \\
\hline $\begin{array}{l}\text { Formeleinheiten je } \\
\text { Elementarzelle }\end{array}$ & 4 & 4 & 4 & 6 & 1 \\
\hline Temperatur & $180 \mathrm{~K}$ & $200 \mathrm{~K}$ & $190 \mathrm{~K}$ & $190 \mathrm{~K}$ & $190 \mathrm{~K}$ \\
\hline \multirow[t]{6}{*}{ Gitterkonstanten } & $a=1197.8(2) \mathrm{pm}$ & $a=921.9(2) \mathrm{pm}$ & $a=919.9(2) \mathrm{pm}$ & $a=1383.1(2) \mathrm{pm}$ & $a=947.7(2) \mathrm{pm}$ \\
\hline & $b=2510.6(5) \mathrm{pm}$ & $b=2357.9(5) \mathrm{pm}$ & $b=2371.8(5) \mathrm{pm}$ & $b=1383.1(2) \mathrm{pm}$ & $b=1241.1(3) \mathrm{pm}$ \\
\hline & $c=1883.8(4) \mathrm{pm}$ & $c=1952.4(4) \mathrm{pm}$ & $c=1934.8(4) \mathrm{pm}$ & $c=3831.6(6) \mathrm{pm}$ & $c=1364.9(3) \mathrm{pm}$ \\
\hline & $\alpha=90^{\circ}$ & $\alpha=90^{\circ}$ & $\alpha=90^{\circ}$ & $\alpha=90^{\circ}$ & $\alpha=95.94(3)^{\circ}$ \\
\hline & $\beta=103.12(3)^{\circ}$ & $\beta=95.38(3)^{\circ}$ & $\beta=94.89(3)^{\circ}$ & $\beta=90^{\circ}$ & $\beta=103.27(3)^{\circ}$ \\
\hline & $\gamma=90^{\circ}$ & $\gamma=90^{\circ}$ & $\gamma=90^{\circ}$ & $\gamma=120^{\circ}$ & $\gamma=93.50(3)^{\circ}$ \\
\hline Volumen & $5517(2) \AA^{3}$ & $4226(2) \AA^{3}$ & $4206(1) \AA^{3}$ & $6347.5(5) \AA^{3}$ & $1548.1(5) \AA^{3}$ \\
\hline Dichte & $1.215 \mathrm{~g} / \mathrm{cm}^{3}$ & $1.420 \mathrm{~g} / \mathrm{cm}^{3}$ & $1.702 \mathrm{~g} / \mathrm{cm}^{3}$ & $1.950 \mathrm{~g} / \mathrm{cm}^{3}$ & $1.868 \mathrm{~g} / \mathrm{cm}^{3}$ \\
\hline Messbereich in $2 \Theta$ & $3-52^{\circ}$ & $3-45^{\circ}$ & $3-52^{\circ}$ & $3-52^{\circ}$ & $3-46^{\circ}$ \\
\hline Gemessene Reflexe & 18395 & 19016 & 28947 & 8958 & 8049 \\
\hline Unabhängige Reflexe & $7936\left(R_{\mathrm{int}}=0.0354\right)$ & $5493\left(R_{\text {int }}=0.1056\right)$ & $7767\left(R_{\mathrm{int}}=0.0459\right)$ & $2658\left(R_{\mathrm{int}}=0.0417\right)$ & $4096\left(R_{\mathrm{int}}=0.0636\right)$ \\
\hline $\begin{array}{l}\text { Unabhängige Reflexe mit } \\
F_{0}>4 \sigma\left(F_{0}\right)\end{array}$ & 6552 & 4880 & 6165 & 2460 & 3580 \\
\hline Parameter & 505 & 361 & 361 & 103 & 220 \\
\hline$\mu\left(\mathrm{Mo}_{\mathrm{K \alpha} \alpha}\right)$ & $1.428 \mathrm{~mm}^{-1}$ & $1.420 \mathrm{~mm}^{-1}$ & $8.683 \mathrm{~mm}^{-1}$ & $12.854 \mathrm{~mm}^{-1}$ & $11.735 \mathrm{~mm}^{-1}$ \\
\hline$R 1$ & 0.0314 & 0.0550 & 0.0431 & 0.0225 & 0.0473 \\
\hline$w R 2$ (alle Daten) & 0.1005 & 0.1574 & 0.1361 & 0.0526 & 0.1249 \\
\hline Restelektronendichte & $0.303 \mathrm{e} / \AA^{3}$ & $2.777 \mathrm{e} / \AA^{3}$ & $3.911 \mathrm{e} / \AA^{3}$ & $1.091 \mathrm{e} / \AA^{3}$ & $3.465 \mathrm{e} / \AA^{3}$ \\
\hline
\end{tabular}


Synthese und Charakterisierung von binären Molekülverbindungen der Gruppe 15

Elemente mit $\mathbf{P}_{2} \mathrm{As}_{2-}$, $\mathbf{P S b}_{2^{-}}, \mathbf{P B i}_{2^{-}}, \mathbf{P B i}_{3^{-}}$und $\mathbf{P}_{2} \mathbf{B i}_{4}-$ Gerüst.

Synthesis and characterisation of binary Molecular compounds of the group 15

Elements with $\mathbf{P}_{2} \mathrm{As}_{2}, \mathrm{PSb}_{2}, \mathrm{PBi}_{2}, \mathrm{PBi}_{3}$ und $\mathbf{P}_{2} \mathrm{Bi}_{4}$ structures

Carsten von Hänisch*, und Sven Stahl

* PD Dr. C. von Hänisch

Institut für Nanotechnologie, Forschungszentrum Karlsruhe

Postfach 3640, D-76021 Karlsruhe

Telefax: 07247/82-6368

E-Mail: carsten.vonhaenisch@int.fzk.de

Bei der Redaktion eingegangen am

Keywords: Arsenic, Antimony, Bismuth, Phosphorus, Ring Compounds

\begin{abstract}
The reactions of $t \mathrm{BuPh}_{2} \mathrm{SiPLi}_{2}$ with $\left(\mathrm{Me}_{3} \mathrm{Si}_{2}\right)_{2} \mathrm{CHAsCl}_{2}$ in molar ratio 1:1 yields the compound $\left[\left(\mathrm{SiMe}_{3}\right)_{2} \mathrm{CHAsPSi} t \mathrm{BuPh}_{2}\right]_{2}$ (1). The structure of $\mathbf{1}$ reveals the formation of a $\mathrm{As}_{2} \mathrm{P}_{2}$ ring compound. In contrast, the reactions of $t \mathrm{BuPh}_{2} \mathrm{SiPLi}_{2}$ with $\left(\mathrm{Me}_{3} \mathrm{Si}_{2}\right)_{2} \mathrm{CHSbCl}_{2}$ or $\left(\mathrm{Me}_{3} \mathrm{Si}\right)_{2} \mathrm{CHBiCl}_{2}$ yield the chloro bridged species $\left[t \mathrm{BuPh}_{2} \mathrm{SiP}\left\{\mathrm{SbClCH}\left(\mathrm{SiMe}_{3}\right)_{2}\right\}_{2}\right](2)$ and $\left[t \mathrm{BuPh}_{2} \mathrm{SiP}\left\{\mathrm{BiClCH}\left(\mathrm{SiMe}_{3}\right)_{2}\right\}_{2}\right]$ (3). Starting from $\mathrm{RMe}_{2} \mathrm{SiPLi}_{2}(\mathrm{R}=\mathrm{Thex}, \mathrm{Me})$ the compounds $\left[\mathrm{P}\left\{\mathrm{BiClCH}\left(\mathrm{SiMe}_{3}\right)_{2}\right\}_{3}\right](\mathbf{4})$ and $\left[\mathrm{P}_{2}\left\{\mathrm{BiClCH}\left(\mathrm{SiMe}_{3}\right)_{2}\right\}_{4}\right](\mathbf{5})$ were obtained from the reactions with $\left(\mathrm{Me}_{3} \mathrm{Si}_{2}\right)_{2} \mathrm{CHBiCl}_{2}$. The Compounds $\mathbf{1 - 5}$ were characterised by single crystal X-Ray diffraction, elemental analysis as well as IR and NMR techniques.
\end{abstract}

\title{
Einleitung
}

Die Synthese von homonuklearen Ringen und Polycyclen der Gruppe-15-Elemente steht bereits seit langer Zeit im Focus der anorganischen Molekülchemie. ${ }^{[1-3]}$ Dabei konnten verschiedenste Molekülstrukturen realisiert werden. Die Palette reicht von kleinen Ringen wie z. B. $\mathrm{P}_{3} t \mathrm{Bu}_{3}$ [2] über größere Ringe bis zu polcylischen oder käfigartigen Spezies [3]. Unter 
den möglichen binären Verbindungen sind bisher in erster Linie die Phosphor-StickstoffHeterocyclen untersucht [4], während über Heterocyclen aus zwei verschiedenen schwereren Elementen der 5. Gruppe ( $\mathrm{P}, \mathrm{As}, \mathrm{Sb}, \mathrm{Bi})$ vergleichsweise wenig bekannt ist. $\mathrm{Zu}$ den beschriebenen Verbindungen dieses Typs gehört z. B. der Ring $\left(\mathrm{P}_{2} \mathrm{As} t \mathrm{Bu}_{3}\right)$, der bei der Reaktion von $t \mathrm{Bu}_{2} \mathrm{P}_{2} \mathrm{~K}_{2}$ mit $t \mathrm{BuAsCl}_{2}$ gebildet wird [5]. Desweiteren sind die bicyclischen Verbindungen $\left[\mathrm{As}_{2}(\mathrm{PAr})_{2}\right]$ and $\left[\mathrm{Sb}_{2}(\mathrm{PAr})_{2}\right]$ bekannt, die durch die Umsetzung von $\operatorname{ArP}\left(\mathrm{SiMe}_{3}\right) \mathrm{Li}\left(\mathrm{Ar}=2,4,6-(t \mathrm{Bu})_{3} \mathrm{C}_{6} \mathrm{H}_{2}\right)$ mit $\mathrm{Cp}^{*} \mathrm{E}^{\prime} \mathrm{Cl}_{2}\left(\mathrm{E}^{\prime}=\mathrm{As}, \mathrm{Sb}\right)$ erhalten wurden sowie die ebenfalls bicyclischen Verbindungen $\left[(t \mathrm{BuP})_{3} \mathrm{As}\right]_{2}$ und $\left[(t \mathrm{BuP})_{3} \mathrm{Sb}\right]_{2}$ [6]. Darüber hinaus wurden kürzlich die binären Käfige $\left[\mathrm{Sb}_{4}\left(\mathrm{PSiMe}_{2} \mathrm{Thex}\right)_{4}\right]$ und $\left[\mathrm{Sb}_{4}\left(\mathrm{AsSi}_{2} \mathrm{Pr}_{3}\right)_{4}\right]$ sowie das phosphanylsubstituierte Dibismuten [BiP( $\left.\left.\operatorname{Sit} \mathrm{BuPh}_{2}\right)_{2}\right]_{2}$ erhalten [7].

Wir berichten hier über die Synthesen und Kristallstrukturen der cyclischen Verbindung $\left[\left(\mathrm{SiMe}_{3}\right)_{2} \mathrm{CHAsPSit} \mathrm{BuPh}_{2}\right]_{2} \quad$ (1) sowie der chlorverbrückten Spezies $\left[t \mathrm{BuPh}_{2} \mathrm{SiP}\left\{\mathrm{SbClCH}\left(\mathrm{SiMe}_{3}\right)_{2}\right\}_{2}\right] \quad$ (2), $\quad\left[t \mathrm{BuPh}_{2} \mathrm{SiP}\left\{\mathrm{BiClCH}\left(\mathrm{SiMe}_{3}\right)_{2}\right\}_{2}\right] \quad$ (3), $\left[\mathrm{P}\left\{\mathrm{BiClCH}\left(\mathrm{SiMe}_{3}\right)_{2}\right\}_{3}\right]$ (4) und $\left[\mathrm{P}_{2}\left\{\mathrm{BiClCH}\left(\mathrm{SiMe}_{3}\right)_{2}\right\}_{4}\right]$ (5), die bei Reaktionen der Dilithiumphosphandiide $t \mathrm{BuPh}_{2} \mathrm{SiPLi}_{2}$, ThexMe $\mathrm{SiPLi}_{2}$ (Thex $=\mathrm{CMe}_{2} i \mathrm{Pr}$ ) und $\mathrm{Me}_{3} \mathrm{SiPLi}_{2}$ mit $\mathrm{R} * \mathrm{ECl}_{2}\left(\mathrm{E}=\mathrm{As}, \mathrm{Sb}, \mathrm{Bi} ; \mathrm{R}^{*}=\mathrm{CH}\left(\mathrm{SiMe}_{3}\right)_{2}\right)$ erhalten wurden (Schema 1).

Schema 1 Darstellung der Verbindungen 1 - 5

\section{Ergebnisse und Diskussion}

Die Reaktion von $t \mathrm{BuPh}_{2} \mathrm{SiPLi}_{2}$ mit $\left(\mathrm{SiMe}_{3}\right)_{2} \mathrm{CHAsCl}_{2}$ in einem molaren Verhältnis von $1: 1$ führt zur Bildung von Verbindung 1, die durch Kühlen der Reaktionslösung auf $-35^{\circ} \mathrm{C}$ in Form von gelben Kristallen erhalten werden kann. 1 kristallisiert in der monoklinen Raumgruppe $P 2_{1} / n$ und besteht aus einem $\mathrm{P}_{2} \mathrm{As}_{2}$-Vierring mit alternierender Elementfolge [9]. Die Phosphoratome weisen je einen exocyclischen Silylsubstituenten auf, jedes der Arsenatome bindet an einen $\mathrm{CH}\left(\mathrm{SiMe}_{3}\right)_{2}$-Rest. Der $\mathrm{P}_{2} \mathrm{As}_{2}$-Ring ist nicht planar sondern weist mit Faltungswinkeln von $149.2^{\circ}$ (entlang der P-P-Achse) und $122.3^{\circ}$ (entlang der As-AsAchse) eine schmetterlingsartige Faltung auf. Die P-As-Bindungen in 1 sind durchschnittlich 236.5 pm lang, die P-As-P- bzw. As-P-As-Winkel betragen durchschnittlich 81.6 bzw. $83.5^{\circ}$. 
Abbildung 1 Molekülstruktur von 1 im Kristall (40\% Wahrscheinlichkeit für die Schwingungsellipsoide, die Wasserstoffatome sind nicht gezeigt).

Ausgesuchte Bindungslängen/pm und -winkel/ ${ }^{\circ}$ : As(1)-P(1) 237.3(1), As(2)-P(1) 235.3(1), $\operatorname{As}(1)-\mathrm{P}(2)$ 236.7(1), As(2)-P(2) 236.8(1), P(1)-Si(1) 227.8(1), P(2)-Si(2) 227.2(1), As(1)C(33) 202.4(3), As(2)-C(40) 201.2(3); As-P-As 83.47(3) und 83.68(3), P-As-P 81.36(3) und 81.75(3), Si(1)-P(1)-As(1) 104.33(4), Si(1)-P(1)-As(2) 96.63(4), Si(2)-P(2)-As(1) 99.61(4), $\quad \mathrm{Si}(2)-\mathrm{P}(2)-\mathrm{As}(2) \quad$ 108.98(4), $\quad \mathrm{C}(33)-\mathrm{As}(1)-\mathrm{P}(1) \quad 110.28(9), \quad \mathrm{C}(33)-\mathrm{As}(1)-\mathrm{P}(2)$ 103.05(9), C(40)-As(2)-P(1) 103.66(9), C(40)-As(2)-P(2) 109.28(7).

Das ${ }^{31}$ P-NMR-Spektrum von 1 zeigt ein Tripplet bei -97 ppm mit einer Kopplungskonstanten von $8 \mathrm{~Hz}$ zu den tertiären Protonen der $\mathrm{CH}\left(\mathrm{SiMe}_{3}\right)_{2}$-Gruppen. Im EI-Massenspektrum von 1 wird der Molekülpeak sowie die Fragmentierung unter Abspaltung der $\mathrm{Sit} \mathrm{BuPh}_{2}$ und der $\mathrm{CH}\left(\mathrm{SiMe}_{3}\right)_{2}$-Substituenten beobachtet.

Überraschend führt die Reaktion von $t \mathrm{BuPh}_{2} \mathrm{SiPLi}_{2}$ mit $\mathrm{R} * \mathrm{SbCl}_{2}\left(\mathrm{R}^{*}=\mathrm{CH}\left(\mathrm{SiMe}_{3}\right)_{2}\right)$ nicht zu einer Ringverbindungen analog $\mathrm{zu} \mathbf{1}$, stattdessen wird die gelbe Verbindung $\left[t \mathrm{BuPh}_{2} \mathrm{SiP}\left\{\mathrm{SbClCH}\left(\mathrm{SiMe}_{3}\right)_{2}\right\}_{2}\right]$ (2) erhalten. Verbindung 2 kristallisiert in der Raumgruppe $P 2_{1} / c$. Die Molekülstruktur wird von einem $t \mathrm{BuPh}_{2} \mathrm{SiP}_{-F r a g m e n t}$ und zwei $\mathrm{Sb}(\mathrm{Cl}) \mathrm{CH}\left(\mathrm{SiMe}_{3}\right)_{2}$-Gruppen aufgebaut. Eines der Cl-Atome koordiniert an das benachbarte Metallatom und befindet sich so in einer stark asymmetrischen Brückenposition. Das Verhältnis von der langen zu der kurzen Sb-Cl-Bindung innerhalb dieser Brücken beträgt 1.39. Der zweite Chloroligand zeigt keine sekundären Wechselwirkungen, weder intra- noch intermolekular. Das $\mathrm{Sb}_{2} \mathrm{P}$-Fragment in $\mathbf{2}$ bildet einen Winkel von $99.4^{\circ}$, die siliziumorganischen Substituenten an den Antimonatomen zeigen eine cis-Orientierung zueinander, der $\mathrm{Si}_{\mathrm{BuPh}}$-Substituent am Phosphoratom steht dazu trans. Die Sb-PBindungen in 2 liegen mit Werten von 254.4 und 255.3 pm im üblichen Bereich für Einfachbindungen zwischen diesen Elementen [6, 7]. Das ${ }^{31} \mathrm{P}\left\{{ }^{1} \mathrm{H}\right\}$-NMR-Spektrum von 2 zeigt ein Singulett bei $-166.8 \mathrm{ppm}, \mathrm{im}{ }^{29} \mathrm{Si}\left\{{ }^{1} \mathrm{H}\right\}$-NMR werden zwei Dublettsignale für die $\mathrm{SiMe}_{3}$-Gruppen bei 1,6 und 2,8 ppm beobachtet, sowie ein weiteres Dublett des $\mathrm{Sit}_{\mathrm{BuPh}} \mathrm{C}^{-}$ Rests bei 7,9 ppm. 
Abbildung 2 Molekülstruktur von 2 im Kristall (40\% Wahrscheinlichkeit für die Schwingungsellipsoide, die Wasserstoffatome sind nicht gezeigt).

Ausgesuchte Bindungslängen/pm und -winkel/ ${ }^{\circ}: \operatorname{Sb}(1)-\mathrm{P} 254.5(2), \mathrm{Sb}(2)-\mathrm{P} 255.3(2), \mathrm{P}-\mathrm{Si}(1)$ 229.6(2), Sb(1)-Cl(1) 240.1(2), Sb(2)-Cl(2) 239.4(2), Sb(1)-Cl(2) 331.7(2), Sb(1)-C(17) 218.6(6), Sb(2)-C(24) 216.9(6); Si(1)-P-Sb(1) 99.69(7), Si(1)-P-Sb(2) 95.78(7), Sb(1)-P$\mathrm{Sb}(2)$ 99.39(6), $\mathrm{P}-\mathrm{Sb}(1)-\mathrm{Cl}(1)$ 95.57(7), $\mathrm{P}-\mathrm{Sb}(2)-\mathrm{Cl}(2)$ 96.89(6).

Die zu 2 analoge Bismutverbindung $\left[t \mathrm{BuPh}_{2} \mathrm{SiP}\left\{\mathrm{BiClCH}\left(\mathrm{SiMe}_{3}\right)_{2}\right\}_{2}\right]$ (3) kann durch Umsetzung von $t \mathrm{BuPh}_{2} \mathrm{SiPLi}_{2}$ mit $\mathrm{R} * \mathrm{BiCl}_{2}$ in Diethylether erhalten werden, sofern bei dieser Reaktion die Temperatur des Reaktionsgemischs nicht auf Werte oberhalb von $-20^{\circ} \mathrm{C}$ steigt. Verbindung 3 kristallisiert isotyp zu 2 und weist bezüglich der Bindungslängen innerhalb der Cl-Brücke ein Verhältnis von 1.25 auf. Die Bi-P-Bi-Gruppe bildet einen Winkel von 96.7 die Bi-P-Bindungen in 3 liegen mit Werten von 262.6 und 265.1 pm im üblichen Bereich für Einfachbindungen zwischen diesen Elementen. So betragen die Bi-P-Bindungslängen beispielsweise in dem oben genannten phosphorsubstituierten Dibismuten $\left[\mathrm{BiP}\left(\operatorname{Si} t \mathrm{BuPh}_{2}\right)_{2}\right]_{2}$ 264.8 pm [7]. Die kristallene Verbindung 3 ist unter Luftausschluss über längere Zeit stabil, in Lösung zersetzt sich $\mathbf{3}$ hingegen schnell unter Bildung der Verbindungen $\mathbf{4}$ und $\mathbf{5}$, elementarem Bismut und weiterer Abbauprodukte.

Abbildung 3 Molekülstruktur von 3 im Kristall (40\% Wahrscheinlichkeit für die Schwingungsellipsoide, die Wasserstoffatome sind nicht gezeigt).

Ausgesuchte Bindungslängen/pm und -winkel/ ${ }^{\circ}$ : Bi(1)-P 262.6(2), Bi(2)-P 265.1(2), P-Si(1) 228.6(3), $\mathrm{Bi}(1)-\mathrm{Cl}(1)$ 252.4(2), $\mathrm{Bi}(2)-\mathrm{Cl}(2)$ 253.3(2), Bi(1)-Cl(2) 316.5(5), Bi(1)-C(17) 228.8(8), Bi(2)-C(24) 228.7(7); Si(1)-P-Bi(1) 95.86(8), Si(1)-P-Bi(2) 98.48(8), Bi(1)-P$\mathrm{Bi}(2)$ 96.66(6), $\mathrm{P}-\mathrm{Bi}(1)-\mathrm{Cl}(1)$ 94.03(7), $\mathrm{P}-\mathrm{Bi}(2)-\mathrm{Cl}(2)$ 95.00(8).

Werden an Stelle von $t \mathrm{BuPh}_{2} \mathrm{SiPLi}_{2}$ die Dilithiumphosphandiide $\mathrm{ThexMe}_{2} \mathrm{SiPLi}_{2}$ oder $\mathrm{Me}_{3} \mathrm{SiPLi}_{2}$ mit $\mathrm{R} * \mathrm{BiCl}_{2}$ in Ether bei $-50^{\circ} \mathrm{C}$ umgesetzt und die Reaktionsgemische anschließend auf Raumtemperatur erwärmt so bilden sich dunkle Lösungen, aus denen rote Kristalle der Verbindungen $\left[\mathrm{P}\left\{\mathrm{BiClCH}\left(\mathrm{SiMe}_{3}\right)_{2}\right\}_{3}\right]$ (4) bzw. $\left[\mathrm{P}_{2}\left\{\mathrm{BiClCH}\left(\mathrm{SiMe}_{3}\right)_{2}\right\}_{4}\right]$ (5) 
isoliert werden können. Die beiden Verbindungen $\mathbf{4}$ und $\mathbf{5}$ sind anders als die Verbindungen $\mathbf{1}$ bis 3 in gängigen organischen Lösungsmitteln weitgehend unlöslich und neigen bei Erwärmung zur Zersetzung unter Abscheidung von elementarem Bismut. Eine NMRspektroskopische Untersuchung dieser beiden Verbindungen in Lösung war infolgedessen nicht möglich.

Bei der Synthese von Verbindung 4 ist es zur vollständigen Substitution sowohl der Lithiumkationen als auch des $\mathrm{SiMe}_{2}$ Thex-Restes durch $\mathrm{BiR}{ }^{*} \mathrm{Cl}-$ Gruppen gekommen. Das $\mathrm{PBi}_{3}$-Fragment weist eine für tertiäre Phosphane typische pyramidale Struktur auf, die Bi-PBi-Winkel betragen $97.0^{\circ}$. Wie in Verbindung $\mathbf{3}$ weisen die Cl-Substituenten eine asymmetrisch verbrückende Position zwischen den Bi-Atomen auf. Das Verhältnis von langer zu kurzer Bi-Cl-Bindung beträgt in dieser Verbindung 1.21. Zusätzlich bilden sich im Kristall schwache intermolekulare Bi-Cl-Bindungen mit einer Länge von 349.1 pm aus, indem sich benachbarte $\mathrm{BiCl}$-Gruppen unter Ausbildung von rautenförmigen $\mathrm{Bi}_{2} \mathrm{Cl}_{2}$-Ringen einander annähern. Dies führt zu einer Vernetzung der Moleküle senkrecht zur kristallographischen cAchse (siehe Abb. 5). Aufgrund dieser Vernetzung und der damit verbundenen höheren Koordinationszahlen an den $\mathrm{Bi}$ - und $\mathrm{Cl}$-Atomen sind die kurzen Bi-Cl-Bindungen in $\mathbf{4}$ mit 263.0 pm um ca. 10 pm länger als die entsprechenden Bindungen in Verbindung 3, bei der keine intermolekularen Wechselwirkungen im Kristall beobachtet werden. Ähnliche interund intramolekulare Bi-Cl-Bindungslängen werden auch in metallorganisch substituierten Bismutchloriden beobachtet: So sind diese Bindungen beispielsweise in der Verbindung $\left[\mathrm{C}_{5} \mathrm{H}_{3} t \mathrm{Bu}_{2}(\mathrm{CO})_{2} \mathrm{FeBiCl}_{2}\right.$ ] zwischen 260.5 und 324.5 pm lang [8].

Abbildung 4 Molekülstruktur von 4 im Kristall $(50 \%$ Wahrscheinlichkeit für die Schwingungsellipsoide, die Wasserstoffatome sind nicht gezeigt).

Ausgesuchte Bindungslängen $/ \mathrm{pm}$ und -winkel/ ${ }^{\circ}$ : Bi-P 263.2(1), Bi-Cl 263.0(1), Bi-Cl 317.0(1), Bi-C(1) 228.6(4); Bi-P-Bi`97.00(5), Bi-Cl-Bi“ 85.09(8). 
Abbildung 5 Netzstruktur von Verbindung 4 durch intermolekulare Bi-ClWechselwirkungen. Von den $\mathrm{CH}\left(\mathrm{SiMe}_{3}\right)_{2}$-Substituenten sind nur die $\alpha$-Kohlenstoffatome abgebildet.

Die Kristallstrukturanalyse von Verbindung 5 erfolgt in der triklinen Raumgruppe $P-1$ und zeigt, dass es zur Abspaltung der Silylgruppen am Phosphor und zur Knüpfung einer P-PBindung gekommen ist. Die entstandene $\mathrm{P}_{2}$-Einheit bindet an vier Bi-Atome, die ihrerseits an je einen $\mathrm{CH}\left(\mathrm{SiMe}_{3}\right)_{2}$-Rest gebunden sind und durch verbrückende Chloroliganden miteinander verknüpft sind. Damit hat das Molekül die Gestalt einer von einem $\mathrm{Bi}_{4} \mathrm{Cl}_{4}$-Ring umgebenen $\mathrm{P}_{2}$-Einheit. Anders als in den Bismutverbindungen 3 und $\mathbf{4}$ sind die Cl-Brücken in 5 weitgehend symmetrisch: Die Bi-Cl-Bindungslängen liegen alle im Bereich von 268.0 bis 287.6 pm. Der $\mathrm{Bi}_{4} \mathrm{Cl}_{4}$-Ring ist praktisch planar, die $\mathrm{P}_{2}$-Einheit liegt hingegen nicht in dieser Ebene, sondern ist aus dieser um einen Winkel von ca. 54 ${ }^{\circ}$ herausgedreht (siehe Abbildung 7). Infolge dieser Verkippung der $\mathrm{P}_{2}$-Einheit gegenüber dem Ring betragen an den Phosphoratomen alle Winkel nahezu $90^{\circ}$.

Abbildung 6 Molekülstruktur von 5 im Kristall (40\% Wahrscheinlichkeit für die Schwingungsellipsoide, die Wasserstoffatome sind nicht gezeigt).

Ausgesuchte Bindungslängen/pm und -winkel/ ${ }^{\circ}$ : Bi(1)-P 265.9(3), Bi(2)-P 265.2(3), P-P` 222.6(7), Bi(1)-Cl(1) 268.0(4), Bi(1)-Cl(2) 287.6(4), Bi(2)-Cl(2) 266.9(3), Bi(2)-Cl(1') 285.3(4); P`-P-Bi(1) 92.1(1), P`-P-Bi(2) 92.8(2), Bi(1)-P-Bi(2) 92.3(1), Bi(1)-Cl(1)-Bi(2`) 105.8(1), $\quad \mathrm{Bi}(1)-\mathrm{Cl}(2)-\mathrm{Bi}(2) \quad 86.7(1), \quad \mathrm{Cl}(1)-\mathrm{Bi}(1)-\mathrm{Cl}(2) \quad 172.1(1), \quad \mathrm{Cl}\left(1^{\prime}\right)-\mathrm{Bi}(2)-\mathrm{Cl}(2)$ 173.6(1).

Abbildung 7 Molekülstruktur von 5 im Kristall (Seitenansicht)

\section{Zusammenfassung und Ausblick}


Die Reaktion des Dilithiumphosphandiids $t \mathrm{BuPh}_{2} \mathrm{SiPLi}_{2}$ mit dem Arsenchlorid $\mathrm{R}^{*} \mathrm{AsCl}_{2}\left(\mathrm{R}^{*}\right.$ $\left.=\mathrm{CH}\left(\mathrm{SiMe}_{3}\right)_{2}\right)$ führt zu der cyclischen Verbindung 1 mit einem zentralen $\mathrm{As}_{2} \mathrm{P}_{2}$-Ring. Hingegen führen Reaktionen desselben Phosphandiids mit den analogen Chloriden der schwereren Homologen ( $\mathrm{Sb}$ und $\mathrm{Bi}$ ) - auch bei einer Durchführung in einem 1:1-Verhähtnis der Ausgangsverbindungen - zu den chloroverbrückten Spezies 2 und 3. Dies kann auf die stabilisierende Wirkung der Chlorobrücken zurückgeführt werden, die bei Arsen nicht in gleichem Maße zu erwarten ist. Werden Dilithiumsilylphosphandiide mit sterisch weniger anspruchsvollen Silylsubstituenten mit $\mathrm{R} * \mathrm{BiCl}_{2}$ umgesetzt, so beobachtet man die Abspaltung der Silylreste, die vermutlich zu den entsprechenden Chlorsilanen umgesetzt werden. Im Fall des ThexMe $\mathrm{S}_{2} \mathrm{Si}$-substituierten Phosphandiids führt dies zur Ausbildung eines Tribismutylphosphans (Verbindung 4), während bei Verwendung des $\mathrm{Me}_{3} \mathrm{Si}$-Substituenten, in folge von Redoxprozessen ein entsprechend substituiertes Diphosphan entsteht (Verbindung 5) Diese oxidative Phosphor-Phosphor-Bindungsknüpfung geht einher mit der Bildung von elementarem Bismut, so das die Vermutung nahe liegt, dass das eingesetzte $\mathrm{R} * \mathrm{BiCl}_{2}$ hier als oxidationsmittel wirkt.

Insbesondere die ungewöhnlichen Verbindungen 4 und 5, die als die ersten zwei Glieder einer homologen Reihe von vollständig bismutylsubstituierten Phosphanen aufgefasst werden können, zeigen, dass auf diesem noch weitgehend unerforschten Gebiet der molekularen binären Verbindungen der Gruppe-15-Elemente noch so manche Überraschung zu erwarten ist. Gegenstand laufender Untersuchungen ist die Synthese weiterer Molekülverbindungen mit $\mathrm{P}-\mathrm{Sb}$ - und P-Bi-Bindungen sowie von Verbindungen der Elementkombinationen As/Sb und $\mathrm{As} / \mathrm{Bi}$.

\section{Experimentelles}

Alle Arbeiten wurden unter Ausschluss von Luft und Feuchtigkeit in einer Atmosphäre von gereinigtem Stickstoff ausgeführt. Die verwendeten Lösungsmittel wurden getrocknet und vor Benutzung frisch destilliert. Die NMR-Spektren wurden an einem Gerät vom Typ DPX Avance 300 der Firma Bruker aufgenommen. $t \mathrm{BuPh}_{2} \mathrm{SiPH}_{2}$, ThexMe $\mathrm{SiPH}_{2}$ und $\mathrm{Me}_{3} \mathrm{SiPH}_{2}$ sowie die Verbindungen $\left(\mathrm{Me}_{3} \mathrm{Si}\right)_{2} \mathrm{CHMCl}_{2}(\mathrm{M}=\mathrm{As}, \mathrm{Sb}, \mathrm{Bi})$ wurden nach bekannten Literaturvorschriften synthetisiert [7b, 10]. 
1: $1.04 \mathrm{~g} \mathrm{(3.82} \mathrm{mmol)} t \mathrm{BuPh}_{2} \mathrm{SiPH}_{2}$ werden in $30 \mathrm{ml}$ Diethylether gelöst und auf $0^{\circ} \mathrm{C}$ gekühlt. $\mathrm{Zu}$ dieser Lösung werden langsam $4.8 \mathrm{ml} 1.6 \mathrm{M}$ (7.64 mmol) $n$ BuLi-Lösung zugetropft. Nach beendeter Zugabe wird die Lösung auf Raumtemperatur erwärmt und weitere 30 Minuten gerührt. Daraufhin wird das Reaktionsgemisch auf $-30{ }^{\circ} \mathrm{C}$ gekühlt und eine Lösung von 1.17 g (3.82 mmol) $\mathrm{R}^{*} \mathrm{AsCl}_{2}\left(\mathrm{R}^{*}=\mathrm{CH}\left(\mathrm{SiMe}_{3}\right)_{2}\right)$ gelöst in $10 \mathrm{ml}$ Diethylether zugegeben. Anschließend wird das Reaktionsgemisch auf Raumtemperatur erwärmt und weitere 16 Stunden gerührt. Nach dem Entfernen des Lösungsmittels wird der verbliebene gelbe Feststoff in Pentan aufgenommen. Nach Filtration fallen aus dieser Lösung bei $-30{ }^{\circ} \mathrm{C}$ gelbe Kristalle der Verbindung 1 aus. Ausbeute: $2.60 \mathrm{~g}$ (68\%). Elementaranalyse (\%) berechnet für: $\mathrm{C}_{46} \mathrm{H}_{76} \mathrm{As}_{2} \mathrm{P}_{2} \mathrm{Si}_{6}$ (1009.40): C 54.73, H 7.59; gefunden: C 54,48 H 7.22.

${ }^{1} \mathbf{H}-\mathbf{N M R}\left(\mathrm{C}_{6} \mathrm{D}_{6}, 25{ }^{\circ} \mathrm{C}\right): \delta=-0.02\left(\mathrm{t},{ }^{3} \mathrm{~J}_{\mathrm{P}, \mathrm{H}}=7.9 \mathrm{~Hz}, \mathrm{CH}\left(\mathrm{SiMe}_{3}\right)_{2}, 2 \mathrm{H}\right), 0.41\left(\mathrm{~s},\left(\mathrm{SiCH}_{3}, 36 \mathrm{H}\right)\right.$, $1.30(\mathrm{~s}, t \mathrm{Bu}, 18 \mathrm{H}), 7.29(\mathrm{~m}, \mathrm{Ph}, 12 \mathrm{H}), 7.91(\mathrm{~m}, \mathrm{Ph}, 8 \mathrm{H}) ;{ }^{31} \mathbf{P}\left({ }^{1} \mathbf{H}\right)-\mathbf{N M R}\left(\mathrm{C}_{6} \mathrm{D}_{6}\right): \delta=-97.7(\mathrm{t}$, $\left.{ }^{3} \mathrm{~J}_{\mathrm{P}, \mathrm{H}}=7.9 \mathrm{~Hz}\right) ; \mathbf{M S}\left(\mathrm{EI}, 70 \mathrm{eV}, 160{ }^{\circ} \mathrm{C}\right) \mathrm{m} / \mathrm{z}(\%): 1008(55)\left[\mathrm{M}^{+}\right], 849$ (100) $\left[\mathrm{M}^{+}{ }_{-}\right.$ $\left.\mathrm{CH}\left(\mathrm{SiMe}_{3}\right)_{2}\right], 769(10)\left[\mathrm{M}^{+}-t \mathrm{BuPh}_{2} \mathrm{Si}\right], 595(100)\left[\mathrm{M}^{+}-\mathrm{CH}\left(\mathrm{SiMe}_{3}\right)_{2},-t \mathrm{BuPh}_{2} \mathrm{Si},-\mathrm{Me}\right], 540$ (45) $\left(\mathrm{M}^{+} / 2\right), 239$ (100) $\left[t \mathrm{BuPh}_{2} \mathrm{Si}^{+}\right], 73$ (100) [ $\left.\mathrm{Me}_{3} \mathrm{Si}+\right]$. IR (KBr): 3070(w), 3049(w), 2948(s), 2893(m), 2856(m), 1953(vw), 1897(vw), 1818(vw)1652(w), 1587(w), 1464(m), 1427(m), 1391(w), 1362(w), 1300(vw), 1248(s), 1190(w), 1157(vw), 1101(s), 998(m), 939(vw), 842(vs), 764(m), 736(m), 699(s), 665(m) 603(m), 510(s) 484(s), 404(w).

2: $0.11 \mathrm{~g}(0.41 \mathrm{mmol}) t \mathrm{BuPh}_{2} \mathrm{SiPH}_{2}$ werden in $5 \mathrm{ml}$ Diethylether gelöst und auf $0^{\circ} \mathrm{C}$ gekühlt. $\mathrm{Zu}$ dieser Lösung werden langsam $0.51 \mathrm{ml} 1.6 \mathrm{M}(0.82 \mathrm{mmol}) n$ BuLi-Lösung zugetropft. Nach beendeter Zugabe wird die Lösung auf Raumtemperatur erwärmt und weitere 30 Minuten gerührt. Daraufhin wird das Reaktionsgemisch auf $-50{ }^{\circ} \mathrm{C}$ gekühlt und eine Lösung von $0.29 \mathrm{~g}(0.82 \mathrm{mmol}) \mathrm{R} * \mathrm{SbCl}_{2}\left(\mathrm{R}^{*}=\mathrm{CH}\left(\mathrm{SiMe}_{3}\right)_{2}\right)$ gelöst in $5 \mathrm{ml}$ Diethylether zugegeben. Daraufhin wird das Reaktionsgemisch auf Raumtemperatur erwärmt, weitere zwei Stunden gerührt und das entstandene Lithiumchlorid durch Filtration entfernt. Aus der so erhaltenen klaren gelben Lösung fallen bei $5{ }^{\circ} \mathrm{C}$ gelbe Kristalle der Verbindung 2 aus. Ausbeute: $0.2 \mathrm{~g}$ (54\% bezogen auf Antimon). Elementaranalyse (\%) berechnet für: $\mathrm{C}_{30} \mathrm{H}_{57} \mathrm{Cl}_{2} \mathrm{PSb}_{2} \mathrm{Si}_{5}$ (903.6): C 39.88, H 6.36; gefunden: C 39.42, H 6.74.

${ }^{1} \mathbf{H}-\mathbf{N M R}\left(\right.$ Tol.- $\left.\mathrm{D}_{8}, 25^{\circ} \mathrm{C}\right): \delta=0.22\left(\mathrm{~s},\left(\mathrm{SiCH}_{3}, 18 \mathrm{H}\right), 0.46\left(\mathrm{~s},\left(\mathrm{SiCH}_{3}, 18 \mathrm{H}\right), 1.34(\mathrm{~s}, t \mathrm{Bu}\right.\right.$, 9H), $1.48\left(\mathrm{~d},{ }^{3} \mathrm{~J}_{\mathrm{P}, \mathrm{H}}=2.7 \mathrm{~Hz}, \mathrm{CH}\left(\mathrm{SiMe}_{3}\right)_{2}, 2 \mathrm{H}\right), 7.29$ (m, Ph, 6H), 7.91 (m, Ph, 4H); ${ }^{31} \mathbf{P}-\mathbf{N M R}$ (Tol.-D $8,25^{\circ} \mathrm{C}$ ): $\delta=-165.3$ (s); IR (KBr): 3071(w), 3049(w), 2949(s), 2891(m), 2855(m), 1485(w), 1468(w), 1459(w), 1427(m), 1360(w), 1251(vs), 1192(w), 1100(m), 1007(w), 
990(m), 970(m), 939(w), 837(vs), 764(m), 738(w), 697(s), 661(m), 599(m), 518(w), 509(s), 483(m), 448(w), 408(w).

3: $0.16 \mathrm{~g}$ (0.59 mmol) $t \mathrm{BuPh}_{2} \mathrm{SiPH}_{2}$ werden in $10 \mathrm{ml}$ Diethylether gelöst und auf $-20{ }^{\circ} \mathrm{C}$ gekühlt. $\mathrm{Zu}$ dieser Lösung werden langsam $0.75 \mathrm{ml} 1.6 \mathrm{M}$ (1.2 mmol) nBuLi-Lösung zugetropft. Nach beendeter Zugabe wird die Lösung auf Raumtemperatur erwärmt und weitere 30 Minuten gerührt. Daraufhin wird das Reaktionsgemisch auf $-60{ }^{\circ} \mathrm{C}$ gekühlt und eine Lösung von $0.52 \mathrm{~g}(0.59 \mathrm{mmol}) \mathrm{R}^{*} \mathrm{BiCl}_{2}\left(\mathrm{R}^{*}=\mathrm{CH}\left(\mathrm{SiMe}_{3}\right)_{2}\right)$ gelöst in $10 \mathrm{ml}$ Diethylether zugegeben. Anschließend wird das Reaktionsgemisch langsam bis auf $-20{ }^{\circ} \mathrm{C}$ erwärmt und bei dieser Temperatur zentrifugiert. Die so erhaltene orange Lösung wird bis auf ein Volumen von ca. $3 \mathrm{ml}$ reduziert und auf $-30{ }^{\circ} \mathrm{C}$ gekühlt. Innerhalb von zwei Tagen bilden sich gelbe Kristalle der Verbindung 3. Ausbeute: $0.19 \mathrm{~g}$ (60\% bezogen auf Bismut). Elementaranalyse (\%) berechnet für: $\mathrm{C}_{30} \mathrm{H}_{57} \mathrm{Bi}_{2} \mathrm{Cl}_{2} \mathrm{PSi}_{5}$ (1078.0): C 33.42, H 5.33; gefunden: C 32.84, H 5.10.

${ }^{1} \mathbf{H}-\mathbf{N M R}$ (Tol.-- $\left.8,25{ }^{\circ} \mathrm{C}\right): \delta=0.21\left(\mathrm{~s},\left(\mathrm{SiCH}_{3}, 18 \mathrm{H}\right), 0.51\left(\mathrm{~s},\left(\mathrm{SiCH}_{3}, 18 \mathrm{H}\right), 1.35\right.\right.$ (s, $t \mathrm{Bu}$, 9H), 1.62 (s, br, $\left.\mathrm{CH}\left(\mathrm{SiMe}_{3}\right)_{2}, 2 \mathrm{H}\right), 7.25$ (m, Ph, 6H), 7.84 (m, Ph, 4H); ${ }^{31}$ P-NMR (Tol.-D, $\left.25^{\circ} \mathrm{C}\right): \delta=-158.3(\mathrm{~s})$; IR (KBr): 3069(w), 3049(w), 2947(vs), 2892(s), 2856(m), 1484(w), 1468(m), 1427(s), 1356(w), 1360(m), 1250(vs), 1191(w), 1100(m), 1009(s), 997(s), 943(m), 907(w), 839(vs), 772(m), 737(m), 699(s), 657(s), 596(m), 512(s), 483(s), 449(w), 408(w).

4: $0.10 \mathrm{~g}(0.57 \mathrm{mmol}) \mathrm{ThexMe}_{2} \mathrm{SiPH}_{2}$ werden in $10 \mathrm{ml}$ Diethylether gelöst und auf $0{ }^{\circ} \mathrm{C}$ gekühlt. $\mathrm{Zu}$ dieser Lösung werden langsam $0.71 \mathrm{ml} 1.6 \mathrm{M}$ (1.14 mmol) nBuLi-Lösung zugetropft. Nach beendeter Zugabe wird die Lösung auf Raumtemperatur erwärmt und weitere 30 Minuten gerührt. Daraufhin wird das Reaktionsgemisch auf $-50{ }^{\circ} \mathrm{C}$ gekühlt und eine Lösung von $0.48 \mathrm{~g}(1.1 \mathrm{mmol}) \mathrm{R}^{*} \mathrm{BiCl}_{2}\left(\mathrm{R}^{*}=\mathrm{CH}\left(\mathrm{SiMe}_{3}\right)_{2}\right)$ gelöst in $15 \mathrm{ml}$ Diethylether zugegeben. Anschließend wird das Reaktionsgemisch langsam auf Raumtemperatur erwärmt und nach 20 Stunden von dem entstandenen Niederschlag $(\mathrm{LiCl})$ befreit. Die so erhaltene rote Lösung wird bis auf ein Volumen von $10 \mathrm{ml}$ reduziert und auf $-30{ }^{\circ} \mathrm{C}$ gekühlt. Innerhalb von drei Tagen bilden sich rote sechseckige plättchenförmige Kristalle der Verbindung 4. Ausbeute: $0.12 \mathrm{~g}$ (26\% bezogen auf Bismut). Elementaranalyse (\%) berechnet für: $\mathrm{C}_{21} \mathrm{H}_{57} \mathrm{Bi}_{3} \mathrm{Cl}_{3} \mathrm{PSi}_{6}$ (1242.5): C 20.30 H 4.62; gefunden: C 20.30, H 4.54.

IR (KBr): 2952(s), 2897(m), 1423(w), 1402(w), 1251(vs), 1099(s), 1011(s), 969(w), 942(w), 842(vs), 771(w), 756(w), 688(m), 658(s), 592(m), 463(w). 
5: $0.04 \mathrm{~g}(0.38 \mathrm{mmol}) \mathrm{Me}_{3} \mathrm{SiPH}_{2}$ werden in $10 \mathrm{ml}$ Diethylether gelöst und auf $0{ }^{\circ} \mathrm{C}$ gekühlt. $\mathrm{Zu}$ dieser Lösung werden langsam $0.47 \mathrm{ml} 1.6 \mathrm{M}(0.76 \mathrm{mmol}) n \mathrm{BuLi}-\mathrm{Lösung}$ zugetropft. Nach beendeter Zugabe wird die Lösung auf Raumtemperatur erwärmt und weitere 30 Minuten gerührt. Daraufhin wird das Reaktionsgemisch auf $-50{ }^{\circ} \mathrm{C}$ gekühlt und eine Lösung von $0.45 \mathrm{~g}(1.0 \mathrm{mmol}) \mathrm{R}^{*} \mathrm{BiCl}_{2}\left(\mathrm{R}^{*}=\mathrm{CH}\left(\mathrm{SiMe}_{3}\right)_{2}\right)$ gelöst in $15 \mathrm{ml}$ Diethylether zugegeben. Anschließend wird das Reaktionsgemisch langsam auf Raumtemperatur erwärmt und nach 20 Stunden von dem entstandenen Niederschlag ( $\mathrm{LiCl}$ und $\mathrm{Bi}$ ) befreit. Die so erhaltene rote Lösung wird auf $-30{ }^{\circ} \mathrm{C}$ gekühlt. Innerhalb von drei Tagen bilden sich rote stäbchenförmige Kristalle der Verbindung 5 $\cdot \mathrm{Et}_{2} \mathrm{O}$. Ausbeute: $0.10 \mathrm{~g}$ (30\% bezogen auf Phosphor). Elementaranalyse (\%) berechnet für: $\mathrm{C}_{28} \mathrm{H}_{76} \mathrm{Bi}_{4} \mathrm{Cl}_{4} \mathrm{P}_{2} \mathrm{Si}_{8} \cdot \mathrm{C}_{4} \mathrm{H}_{10} \mathrm{O}$ (1751.4): C $21.95 \mathrm{H}$ 4.95; gefunden: C 21.49, H 4.98.

IR (KBr): 2947(s), 2895(m), 2856(m), 1400(m), 1250(vs), 1191(w), 1099(w), 1009(m), 962(w), 941(w), 841(vs), 771(m), 686(w), 656(m), 590(w), 467(w).

Der Deutschen Forschungsgemeinschaft danken wir für die Förderung dieser Untersuchungen.

\section{Literatur}

[1] Übersichten: a) L. Balázs, H. J. Breunig, Coord. Chem. Rev. 2004, 248, 603-621; b) H. J. Breunig, R. Rösler, Coord. Chem. Rev. 1997, 163, 33-53; c) M. Baudler, Angew. Chem. 1987, 99, 429-451; Angew. Chem. Int. Ed. Engl. 1987, 26, 419-441; d) M. Baudler, Angew. Chem. 1982, 94, 520-539; Angew. Chem. Int. Ed. Engl. 1982, 21, 492512.

[2] a) J. Hahn, M. Baudler, Z. Naturforsch. 1982, 37b, 797-805; b) O. Mundt, G. Becker, H.-J. Wessely, Z. Anorg. Allg. Chem. 1982, 486, 70-89; c) M. Baudler, P. Bachmann, Angew. Chem. 1981, 93, 112-113; Angew. Chem. Int. Ed. Engl. 1981, 20, 123-124.

[3] a) M. Westerhausen, S. Weinrich, P. Mayer, Z. Anorg. Allg. Chem. 2003, 629, 11531156; b) G. Linti, W. Köstler, Z. Anorg. Allg. Chem. 2002, 628, 63-66; c) H. J. Breunig, R. Rösler, E. Lork, Angew. Chem. 1997, 109, 2333-2334; Angew. Chem. Int. Ed. Engl. 1997, 36, 2237-2238; d) C. von Hänisch, D. Fenske, Z. Anorg. Allg. Chem. 1997, 623, 
1040-1042; e) M. Baudler, H. Jachow, B. Lieser, K.-F. Tebbe, M. Fehér, Angew. Chem. 1989, 101, 1245-1247; Angew. Chem. Int. Ed. Engl. 1989, 28, 1231-1232; f) C. Mujica, D. Weber, H.-G. Schnering, Z. Naturforsch. 1986, 41b, 991-999; g) M. Baudler, S. Wietfeldt-Haltenhoff, Angew. Chem. 1985, 97, 986-987; Angew. Chem. Int. Ed. Engl. 1985, 24, 991-992; h) M. Baudler, S. Wietfeldt-Haltenhoff, Angew. Chem. 1984, 96, 361-362; Angew. Chem. Int. Ed. Engl. 1984, 23, 379-380; i) M. Baudler, P. Bachmann, Z. Anorg. Allg. Chem. 1982, 485, 129-132; j) M. Baudler, J. Hellmann, P. Bachmann, K.-F. Tebbe, R. Fröhlich, M. Feher, Angew. Chem. 1981, 91, 415-417; Angew. Chem. Int. Ed. Engl. 1981, 20, 406-407; k) H.-G. von Schnering, D. Fenske, W. Hönle, M. Binnewies, K. Peters, Angew. Chem. 1979, 91, 755-756; Angew. Chem. Int. Ed. Engl. 1979, 18, 679; 1) A. Tzschach, V. Kiesel, J. praktische Chem. 1971, 313, 259-264.

[4] E. L. Doyle, L. Riera, D. S. Wright, Eur. J. Inorg. Chem. 2003, 3279-3289.

[5] a) M. Baudler, S. Klautke, Z. Naturforsch. 1981, 36b, 527-531; b) M. Baudler, D. Habermann, Angew. Chem. 1979, 91, 939-940; Angew. Chem. Int. Ed. Engl. 1979, 18, 877-878.

[6] a) A. Bashall, F. Garcia, G. T. Lawson, M. McPartlin, A. Rothenberger, A. D. Woods, D. S. Wright, Can. J. Chem. 2002, 80, 1421-1427; b) P. Jutzi, U. Meyer, S. Opiela, M. Olmstead, P. P. Power, Organometallics 1990, 9, 1459-1463; c) M. Baudler, Y. Aktalay, T. Heinlein, K.-F. Tebbe, Z. Naturforsch. 1982, 37b, 299-305.

[7] a) C. von Hänisch, D. Nikolova, Eur. J. Inorg. Chem. 2006, 4770-4773; b) C. von Hänisch, D. Nikolova, Eur. J. Inorg. Chem. 2005, 378-382.

[8] a) T. Gröer, M. Scheer, J. Chem. Soc. Dalton Trans. 2000, 647-653; b) W. Clegg, N. A. Compton, R. J. Errington, G. A. Fisher, D. C. R. Hockless, N. C. Norman, N. A. L. Williams, S. E. Statford, S. J. Nichols, P. S. Jarrett, A. G. Orpen, J. Chem. Soc. Dalton Trans. 1992, 193- 201; c) J. M. Wallis, G. Müller, H.Schmidbaur, J. Organomet. Chem. 1987, 325, 159-168.

[9] Die Datensammlungen wurden auf einem Flächendetektor STOE IPDS2 (Graphitmonochromator, Mo- $\mathrm{K}_{\alpha}-$ Strahlung) durchgeführt. Die Strukturlösungen erfolgten mit direkten Methoden, verfeinert gegen $F^{2}$ : (Bi, Sb, As, Cl, P, Si, O, C anisotrop verfeinert, H-Atomlagen wurden berechnet. Verbindung 5 kristallisiert mit einem Molekül Diethylether, dieses wurde mit Splitlagen und ohne Wasserstoffatome verfeinert). Die kristallographischen Daten (ohne Strukturfaktoren) der in dieser Veröffentlichung beschriebenen Strukturen wurden als ,supplementary publication no. CCDC-724315 - 724319“ beim Cambridge Crystallographic Data Centre hinterlegt. 
Kopien der Daten können kostenlos bei folgender Adresse in Großbritannien angefordert werden: CCDC, 12 Union Road, Cambridge CB21EZ (Fax: (+44)1223-336033; E-mail: deposit@ccdc.cam.ac.uk).

[10] a) H. Althaus, H. J. Breunig, R. Rösler, E. Lork, Organometallics, 1999, 18, 328-331; b) G. Fritz, W. Schick, Z. Anorg. Allg. Chem. 1984, 511, 108-131; c) H. J. Breunig, W. Kanig, A. Soltani-Nesham, Polyhedron, 1983, 2, 291-292; d) M. J. S. Gynane, A. Hudson, M. F. Lappert, P. P. Power, H. Goldwhite, J. Chem. Soc. Dalton 1980, 24282433. 
Tabelle 1 Kristallographische Daten der Verbindungen 1-5 [9].

\begin{tabular}{|c|c|c|c|c|c|}
\hline Verbindung & 1 & 2 & 3 & 4 & 5 \\
\hline Formel & $\mathrm{C}_{46} \mathrm{H}_{76} \mathrm{As}_{2} \mathrm{P}_{2} \mathrm{Si}_{6}$ & $\mathrm{C}_{30} \mathrm{H}_{57} \mathrm{Cl}_{2} \mathrm{PSb}_{2} \mathrm{Si}_{5}$ & $\mathrm{C}_{30} \mathrm{H}_{57} \mathrm{Bi}_{2} \mathrm{Cl}_{2} \mathrm{PSi}_{5}$ & $\mathrm{C}_{21} \mathrm{H}_{57} \mathrm{Bi}_{3} \mathrm{Cl}_{3} \mathrm{PSi}_{6}$ & $\begin{array}{l}\mathrm{C}_{28} \mathrm{H}_{76} \mathrm{Bi}_{4} \mathrm{Cl}_{4} \mathrm{P}_{2} \mathrm{Si}_{8} . \\
\mathrm{C}_{4} \mathrm{H}_{10} \mathrm{O}\end{array}$ \\
\hline Raumgruppe & $P 2_{1} / n$ & $P 2{ }_{1} / c$ & $P 2{ }_{1} / c$ & $R-3$ & $P-1$ \\
\hline $\begin{array}{l}\text { Formeleinheiten je } \\
\text { Elementarzelle }\end{array}$ & 4 & 4 & 4 & 6 & 1 \\
\hline Temperatur & $180 \mathrm{~K}$ & $200 \mathrm{~K}$ & $190 \mathrm{~K}$ & $190 \mathrm{~K}$ & $190 \mathrm{~K}$ \\
\hline \multirow[t]{6}{*}{ Gitterkonstanten } & $a=1197.8(2) \mathrm{pm}$ & $a=921.9(2) \mathrm{pm}$ & $a=919.9(2) \mathrm{pm}$ & $a=1383.1(2) \mathrm{pm}$ & $a=947.7(2) \mathrm{pm}$ \\
\hline & $b=2510.6(5) \mathrm{pm}$ & $b=2357.9(5) \mathrm{pm}$ & $b=2371.8(5) \mathrm{pm}$ & $b=1383.1(2) \mathrm{pm}$ & $b=1241.1(3) \mathrm{pm}$ \\
\hline & $c=1883.8(4) \mathrm{pm}$ & $c=1952.4(4) \mathrm{pm}$ & $c=1934.8(4) \mathrm{pm}$ & $c=3831.6(6) \mathrm{pm}$ & $c=1364.9(3) \mathrm{pm}$ \\
\hline & $\alpha=90^{\circ}$ & $\alpha=90^{\circ}$ & $\alpha=90^{\circ}$ & $\alpha=90^{\circ}$ & $\alpha=95.94(3)^{\circ}$ \\
\hline & $\beta=103.12(3)^{\circ}$ & $\beta=95.38(3)^{\circ}$ & $\beta=94.89(3)^{\circ}$ & $\beta=90^{\circ}$ & $\beta=103.27(3)^{\circ}$ \\
\hline & $\gamma=90^{\circ}$ & $\gamma=90^{\circ}$ & $\gamma=90^{\circ}$ & $\gamma=120^{\circ}$ & $\gamma=93.50(3)^{\circ}$ \\
\hline Volumen & $5517(2) \AA^{3}$ & $4226(2) \AA^{3}$ & $4206(1) \AA^{3}$ & $6347.5(5) \AA^{3}$ & $1548.1(5) \AA^{3}$ \\
\hline Dichte & $1.215 \mathrm{~g} / \mathrm{cm}^{3}$ & $1.420 \mathrm{~g} / \mathrm{cm}^{3}$ & $1.702 \mathrm{~g} / \mathrm{cm}^{3}$ & $1.950 \mathrm{~g} / \mathrm{cm}^{3}$ & $1.868 \mathrm{~g} / \mathrm{cm}^{3}$ \\
\hline Messbereich in $2 \Theta$ & $3-52^{\circ}$ & $3-45^{\circ}$ & $3-52^{\circ}$ & $3-52^{\circ}$ & $3-46^{\circ}$ \\
\hline Gemessene Reflexe & 18395 & 19016 & 28947 & 8958 & 8049 \\
\hline Unabhängige Reflexe & $7936\left(R_{\mathrm{int}}=0.0354\right)$ & $5493\left(R_{\text {int }}=0.1056\right)$ & $7767\left(R_{\mathrm{int}}=0.0459\right)$ & $2658\left(R_{\mathrm{int}}=0.0417\right)$ & $4096\left(R_{\mathrm{int}}=0.0636\right)$ \\
\hline $\begin{array}{l}\text { Unabhängige Reflexe mit } \\
F_{0}>4 \sigma\left(F_{0}\right)\end{array}$ & 6552 & 4880 & 6165 & 2460 & 3580 \\
\hline Parameter & 505 & 361 & 361 & 103 & 220 \\
\hline$\mu\left(\mathrm{Mo}_{\mathrm{K} \alpha}\right)$ & $1.428 \mathrm{~mm}^{-1}$ & $1.420 \mathrm{~mm}^{-1}$ & $8.683 \mathrm{~mm}^{-1}$ & $12.854 \mathrm{~mm}^{-1}$ & $11.735 \mathrm{~mm}^{-1}$ \\
\hline$R 1$ & 0.0314 & 0.0550 & 0.0431 & 0.0225 & 0.0473 \\
\hline$w R 2$ (alle Daten) & 0.1005 & 0.1574 & 0.1361 & 0.0526 & 0.1249 \\
\hline Restelektronendichte & $0.303 \mathrm{e} / \AA^{3}$ & $2.777 \mathrm{e} / \AA^{3}$ & $3.911 \mathrm{e} / \AA^{3}$ & $1.091 \mathrm{e} / \AA^{3}$ & $3.465 \mathrm{e} / \AA^{3}$ \\
\hline
\end{tabular}

